\title{
Photochemistry-emission coupled model for Europa and Ganymede
}

\author{
Gaël Cessateur ${ }^{1,2, *}$, Mathieu Barthelemy ${ }^{3}$, and Isabel Peinke ${ }^{3}$ \\ 1 Royal Belgian Institute for Space Aeronomy, Ringlaan-3-Avenue Circulaire, 1180 Brussels, Belgium \\ ${ }^{*}$ Corresponding author: gael.cessateur@aeronomie.be \\ 2 Physikalisch-Meteorologishes Observatorium Davos, World Radiation Center (PMOD/WRC), Dorfstrasse 33, \\ 7260 Davos Dorf, Switzerland \\ 3 UJF-Grenoble 1/CNRS-INSU, Institut de Planétologie et d'Astrophysique de Grenoble (IPAG), UMR 5274, 38041 Grenoble, France
}

Received 26 May 2015 / Accepted 26 January 2016

\section{ABSTRACT}

\begin{abstract}
In the frame of the JUICE mission, preliminary studies of the Jupiter's icy moons, such as Ganymede and Europa, are mandatory. The present paper aims at characterizing the impact of the solar UV flux and its variability on their atmospheres. The solar UV radiation is responsible for the photoionization, photodissociation, and photoexcitation processes within planetary atmospheres. A 1-D photoabsorption model has been developed for different observational geometries, on the basis of a neutral atmospheric model. Considering various production and loss mechanisms but also the transport of oxygen atoms, we estimate the red and green line emissions from photo impact-induced excitation only. These dayglow emissions can represent few percent of the global airglow emission, mainly dominated by electron-induced excitation in auroral regions. For limb viewing conditions, red line emission is bright enough to be detected from actual spectrometers, from $338 \mathrm{R}$ to $408 \mathrm{R}$ according to the solar activity. This is also the case for the green line with $8 \mathrm{R}$ at limb viewing. Considering a different neutral atmosphere model, with an $\mathrm{O}_{2}$ column density $50 \%$ more important, leads to a $14 \%$ increase in the red line emissions for limb viewing close to the surface. This difference could be important enough to infer which neutral model is the most likely. However, uncertainties on the solar UV flux might also prevent to constrain the $\mathrm{O}_{2}$ column density when using ground-based observations in the visible only. The impact of solar flares on the red line emissions for Europa has also been investigated within a planetary space weather context.
\end{abstract}

Key words. Jovian moons - Aeronomy - Airglow - UV flux - Solar activity

\section{Introduction and motivations}

Planetary space weather is currently an area of high scientific interest (Lilensten et al. 2014), especially in the frame of the exploration of planetary environments such as the Jovian icy moons. In the frame of the JUICE (JUpiter ICy moons Explorer) mission, studies of such objects are of particular importance especially to plan instrument performances (Grasset et al. 2013). The scientific incentive for the JUICE mission is to study not only Jupiter, but also Europa and Ganymede, which are the archetype of icy moons. Both planetary objects are of particular interest for their potential habitability. This concept refers to whether environmental conditions are available that could support life and does not relate to whatever life presently exists or has existed on those worlds. JUICE will address this question not only by constraining the volume of water in the Jovian system, but also by realizing an inventory of elements on the surfaces as well as in the atmospheres of the icy moons. The need for accurate space weather studies of the exospheric variability regarding these two moons is therefore reinforced. An important challenge is thus to find the relevant observable quantities to characterize the space weather variations. In this paper, special focus will be given to the various emission and quenching processes within Ganymede's and Europa's atmospheres, regarding different levels of solar activity. As outcomes, several key physical parameters, namely atmospheric emissions from the visible spectral range, will be estimated.

The tenuous atmospheres of both icy moons are examples of surface-bounded atmospheres. Observations, combined with numerous models of the neutral atmospheres for Europa and Ganymede, give valuable information about the complexity of such surface-bounded atmospheres. While Ganymede and Europa share similar characteristics, each of them has its own specific features. Observations from the Hubble Space Telescope (HST) have confirmed the presence of an $\mathrm{O}_{2}$ atmosphere for both icy moons (Hall et al. 1998). The ionosphere has been detected by in-situ observations by Galileo (Gurnett et al. 1996; Kliore et al. 1997). The surface of both moons is widely covered by water ice (Calvin et al. 1995). The main processes responsible for the formation of such exospheres are direct sputtering by heavy ions (e.g. $\mathrm{S}^{+}$) located within Jupiter's strong magnetic field as measured by Galileo (Paranicas et al. 1999). This leads to the release of water molecules which can subsequently get radiolytically dissociated by sputtering resulting in the formation of new molecules (e.g. $\mathrm{O}_{2}$, $\mathrm{H}_{2}$ ), finally ejected from the surface into the exosphere. At Ganymede, sublimation of water molecules has an equivalent contribution to the exosphere generation for equatorial regions where the temperature of the surface is of about $150 \mathrm{~K}$. The solar UV flux also contributes to the exosphere generation through the Photon Stimulated Desorption mechanism, but with an efficiency lower than that due to sputtering about 1.5 orders of magnitude (Plainaki et al. 2010). Based on these physical processes, numerical models show that the exospheres are mainly dominated by $\mathrm{O}_{2}$ and $\mathrm{H}_{2}$ for Europa (Shematovich et al. 2005; Smyth \& Marconi 2006; Plainaki et al. 2013) with an $\mathrm{O}_{2} / \mathrm{H}_{2} \mathrm{O}$ ratio of about 15 at $0.04 R_{E}$ and for polar region for Ganymede (Marconi 2007; Turc et al. 2014) with an $\mathrm{O}_{2} / \mathrm{H}_{2} \mathrm{O}$ ratio of about 130 at $0.04 R_{G}$. For equatorial regions at 
Ganymede, Marconi (2007) proposed an $\mathrm{H}_{2} \mathrm{O}$ - and $\mathrm{H}_{2}$-dominated atmosphere with a very low density of $\mathrm{O}_{2}$ (with an $\mathrm{O}_{2} / \mathrm{H}_{2} \mathrm{O}$ ratio of about $10^{-4}$ at $0.04 R_{G}$ ). Recent numerical models by Plainaki et al. (2015) suggest however an $\mathrm{O}_{2}$ atmosphere more homogeneous for both polar and equatorial regions within the first $100 \mathrm{~km}$ from the surface: the $\mathrm{O}_{2}$ density is up to two orders of magnitude higher than the ones for polar regions derived from Marconi (2007) and Turc et al. (2014) at $0.05 R_{G}$. The water is still the dominant atmospheric species for equatorial regions at Ganymede. This discrepancy is explained by taking the multiple bouncing of the released $\mathrm{O}_{2}$ molecules into account (see e.g. Cassidy et al. 2007). At higher altitudes, however, an inhomogeneous atmosphere is still expected following the inhomogeneity in the surface release, the sublimation being an efficient process at the equator. More detailed observations of Europa's and Ganymede's environment are thus needed in order to provide more accurate atmospheric models.

Ganymede is also unique for its intrinsic magnetic field with a dipole configuration (Kivelson et al. 1996). Interaction with the Jupiter magnetic field leads to open field lines in the polar regions, and closed ones for latitudes below $45^{\circ}$. Precipitating electrons along the open field lines have an impact on the atmospheric $\mathrm{O}_{2}$, which in turn leads to auroral emissions in the UV as observed by the Hubble Space Telescope (HST; Hall et al. 1998; Feldman et al. 2000; McGrath et al. 2013). The observations of the auroral ovals as observed in the UV might also be used to characterize the subsurface ocean (Saur et al. 2015). Numerous modeling studies have showed that electron impact is the dominant dissociation mechanism of $\mathrm{O}_{2}$ (Saur et al. 1998; Eviatar et al. 2001; Payan et al. 2015), leading to the generation of atomic oxygen in the $\mathrm{O}\left({ }^{5} \mathrm{~S}\right)$ and $\mathrm{O}\left({ }^{3} \mathrm{~S}\right)$ states which produce subsequently emission in the UV at 135.6 and $130.4 \mathrm{~nm}$, respectively.

Observations of emissions in the visible are also good parameters for determining the different energetic inputs coming in upper planetary atmospheres, i.e. solar UV photons and precipitating particles, with the green $(557.7 \mathrm{~nm})$ and reddoublet $(630,636.4 \mathrm{~nm})$ lines coming from the deactivation of oxygen atoms in the $\mathrm{O}\left({ }^{1} \mathrm{~S}\right)$ and $\mathrm{O}\left({ }^{1} \mathrm{D}\right)$ states, respectively. These two visible lines have been indeed largely observed in the case of the Earth (see e.g. Witasse et al. 1999; Thirupathaiah \& Singh 2014). More generally, these emissions are direct signatures of the presence of oxygen-bearing atmospheric species, such as $\mathrm{O}_{2}$ or $\mathrm{H}_{2} \mathrm{O}$. Regarding Europa and Ganymede, observations of visible emissions are rather scarce. For Europa, observations in the visible have been reported by Porco et al. (2003): using the ISS narrow angle camera onboard Cassini with a broadband filter (200-1050 nm), emissions with a peak brightness of about $10 \mathrm{kR}$, with noise of the order of $5 \mathrm{kR}$, have been observed. Cassidy et al. (2008) have attributed these emissions majorly to the electron impact-induced excitation of sodium leading to emissions at $589 \mathrm{~nm}\left(\mathrm{Na}\left({ }^{2} \mathrm{~S}\right)-\mathrm{Na}\left({ }^{2} \mathrm{P}\right)\right.$ transition). The contribution of oxygen atoms to these visible emissions, including the red and green lines, is deduced to be limited with brightness values of about $100 \mathrm{R}$, and mostly at $777.4 \mathrm{~nm}$ and $844.6 \mathrm{~nm}$ from $\mathrm{O}\left({ }^{5} \mathrm{P}\right)-\mathrm{O}\left({ }^{5} \mathrm{~S}\right)$ and $\mathrm{O}\left({ }^{3} \mathrm{P}\right)$ $\mathrm{O}\left({ }^{3} \mathrm{~S}\right)$ transitions, respectively. Moreover, no emissions have been reported for Ganymede during the same Cassini observation campaigns. The relative importance of photochemical processes compared to electronic impact processes on the visible dayglow emissions has never been properly estimated for Europa and Ganymede. Electron impact is the dominant dissociation mechanism, but photodissociation is an important loss process and an important supply of oxygen to the neutral torus, potentially including oxygen atoms in the $\mathrm{O}\left({ }^{1} \mathrm{~S}\right)$ and $\mathrm{O}\left({ }^{1} \mathrm{D}\right)$ states, as discussed by Shematovich et al. (2005).

Observations of these visible emissions can help to constrain neutral atmospheric profiles. However, limb in-situ observations from an orbiter are necessary for measuring the green and red-doublet emissions. Earth-based observations provide only limited ability (e.g. with HST observations; Roth et al. 2014) to look at the vertical profile of the different volatile species of Europa and Ganymede or at the variation with latitude. Indeed, Earth-based observations suffer from too much scattered light. The first motivation of this paper is to provide some emission predictions to support Europa and Ganymede observations in the frame of the JUICE mission, by considering the photo impact-induced excitation only.

In a more general space weather frame, the second motivation is following Barthelemy \& Cessateur (2014) studies on the solar UV flux accuracy to demonstrate on a case study, i.e. Europa and Ganymede, how the knowledge of the solar UV flux is of primary importance regarding the visible red and green dayglow emission estimations especially in the case of solar eruptive events. Since those estimations are directly linked to both the neutral atmospheric model of the planetary object and the solar UV flux, a flawed knowledge of the last one leads to an important uncertainty in the deduced atmospheric compositions. Adding to this, estimations of photochemical processes are relevant in the framework of the JUICE mission to properly disentangle the origin of potential detection of red and green line emissions.

The paper is organized as follows. In Section 2, the photolysis model within Europa's atmosphere is discussed, using two different solar activity levels as well as different observational geometries. In Section 3, dayglow emission for the red and green lines will be estimated based on several production and loss mechanisms of the atomic $\mathrm{O}\left({ }^{1} \mathrm{D}\right)$ and $\mathrm{O}\left({ }^{1} \mathrm{~S}\right)$ states and by taking the transport of neutral oxygen atoms into account. The term dayglow is rather ambiguous since it can refer to the atmospheric emissions due to both solar UV photons and particle precipitations. In this paper, we consider the term dayglow referring to atmospheric emissions from photo impact-induced only. In Section 4, estimations of the red and green line dayglow for Ganymede will also be presented. Dayglow emission for Ganymede will be compared to auroral emissions in order to estimate the contribution of photon-induced to electron-induced emissions in Section 5. The importance of the chosen solar UV flux for dayglow modeling is discussed in Section 6, and a summary is given in Section 7.

\section{Photolysis in Europa's atmosphere}

\subsection{Neutral atmospheric models}

Two neutral atmospheric models will be used throughout this paper, the model of Smyth \& Marconi (2006) and the one of Shematovich et al. (2005) (hereafter named Models 1 and 2, respectively):

- Model 1 from Smyth \& Marconi (2006) is a two-dimensional kinetic model providing the density profiles for the water group species $\left(\mathrm{H}_{2} \mathrm{O}, \mathrm{OH}, \mathrm{O}_{2}, \mathrm{O}, \mathrm{H}_{2}\right.$, and $\left.\mathrm{H}\right)$. Europa's atmosphere is globally dominated by $\mathrm{O}_{2}$ at lower altitudes, and by $\mathrm{H}_{2}$ at higher altitudes, as illustrated in Figure 1. 
G. Cessateur et al.: Photochemistry-emission coupled model for Europa and Ganymede

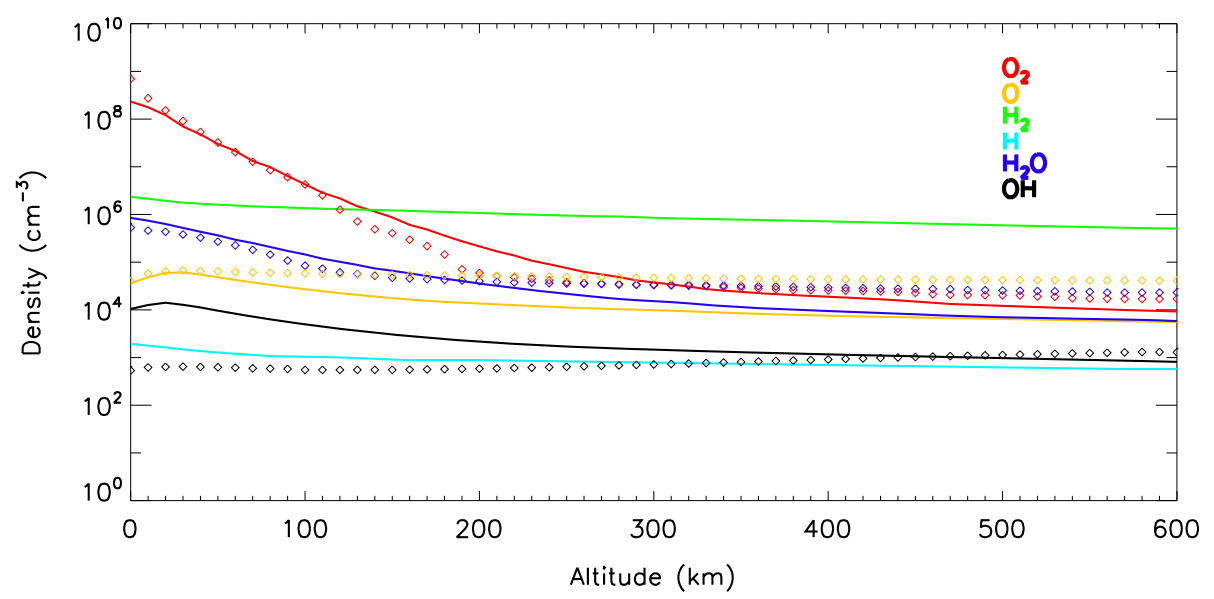

Fig. 1. Radial density profiles for the water group species of Europa's atmosphere of Smyth \& Marconi (2006) (thick lines) and of Shematovich et al. (2005) (dotted lines).

- Model 2 is taken from Shematovich et al. (2005), who proposed a series of 1-D collisional kinetic models which differ in the initial $\mathrm{O}_{2}$ and $\mathrm{H}_{2}$ source rates and loss processes considered. Among these different models, we choose Model $\mathrm{F}$, in which the $\mathrm{O}_{2}$ surface source rate depends only on sputtering processes, while the $\mathrm{H}_{2} \mathrm{O}$ surface source rate depends both on sputtering and sublimation processes (with a surface temperature of about $100 \mathrm{~K}$ ).

The key difference between these two neutral atmospheric models lies in the $\mathrm{O}_{2}$ and $\mathrm{O}$ vertical profiles. In terms of column density, Model 2 has greater values compared to Model 1 with an increase of about $50 \%$ and $290 \%$, respectively. This $\mathrm{O}_{2}$ enrichment takes place for altitudes below $50 \mathrm{~km}$. Model 2 does not provide the density profiles for $\mathrm{H}_{2}$ and $\mathrm{H}$. Comparison between atmospheric models will thus be performed when focusing on the processes involving $\mathrm{O}_{2}$ and $\mathrm{O}$, namely dayglow emission of atomic oxygen (see Sect. 3.3). The column densities estimated on the basis of Models 1 and 2 are also shown in Table 1. Let us also note that these column densities are higher compared to the ones presented by Smyth \& Marconi (2006) and Shematovich et al. (2005). For numerical reasons, both atmospheric models have been interpolated till the ground, which leads to an increase in the column density values. It is important to mention that the following results regarding both photoionization and photoexcitation processes within Europa's atmosphere are only related to the column density values provided in Table 1, and not to the values provided by Smyth \& Marconi (2006) and Shematovich et al. (2005).

\subsection{Atmospheric response to solar UV flux}

Four different cases with the geometries shown in Figure 2 are considered, where a spherical symmetry regarding the atmosphere is assumed. Case 1 represents the spacecraft above the equator looking to the nadir with a zero solar zenith angle. Case 2 corresponds to a nadir view at a latitude of $82^{\circ}$. Cases 3 and 4 simulate the observation of an orbiter which would look $10 \mathrm{~km}$ and $100 \mathrm{~km}$ above the surface of the planet, respectively. The line of sight crosses the whole atmosphere from beginning to end, with the closest altitude regarding Europa's surface being $10 \mathrm{~km}$ and $100 \mathrm{~km}$. A line of sight abscissa is defined as the distance from the tangent point along the line of sight. The top of the atmosphere, closer to the observing
Table 1. Column density (in $\mathrm{cm}^{-2}$ ) for the considered species based on both neutral atmospheric models.

\begin{tabular}{lcc}
\hline \hline Species & $\begin{array}{c}\text { Model 1 (Smyth \& } \\
\text { Marconi 2006) }\end{array}$ & $\begin{array}{c}\text { Model 2 (Shematovich } \\
\text { et al. 2005) }\end{array}$ \\
\hline $\mathrm{O}_{2}$ & $6.2 \times 10^{14}$ & $9.48 \times 10^{14}$ \\
$\mathrm{H}_{2}$ & $7.4 \times 10^{13}$ & - \\
$\mathrm{H}_{2} \mathrm{O}$ & $5.5 \times 10^{12}$ & $5.48 \times 10^{12}$ \\
$\mathrm{OH}$ & $2.03 \times 10^{11}$ & $8.96 \times 10^{10}$ \\
$\mathrm{O}$ & $1.14 \times 10^{12}$ & $4.48 \times 10^{12}$ \\
$\mathrm{H}$ & $6.99 \times 10^{10}$ & - \\
\hline
\end{tabular}

orbiter, is $2022 \mathrm{~km}(1920 \mathrm{~km}$ in case 4) and -2022 km (resp. $-1920 \mathrm{~km}$ ) for the farther point (corresponding to an altitude of $1000 \mathrm{~km}$ relative to Europa's surface). The atmosphere has been projected along the line of sight. The altitude regarding Europa's surface is calculated for each point of the line of sight, and the corresponding atmosphere is attributed.

Photoionization processes mainly involve the extreme UV (EUV, 10-121 nm), while photoexcitation and photodissociation processes involve the far UV (FUV, 122-200 nm) and middle UV (MUV, 200-300 nm). The solar UV flux as measured by recent space missions will be first used in order to estimate dayglow emission. Two different solar activities are considered, a moderate one $(\mathrm{F} 10.7=83)$ and a strong one $(\mathrm{F} 10.7=195)$. The data in the $27-115 \mathrm{~nm}$ range is measured by the EUV Grating Spectrograph (EGS), which is part of the Solar Extreme Ultraviolet Experiment (SEE) onboard TIMED (Woods et al. 2005). The 115-250 nm range is covered by the SOLar Stellar Irradiance Comparison Experiment (SOLSTICE) instrument onboard the SOLar Radiation and Climate Experiment (SORCE; Rottman 2005). Data in the 1-27 nm range come from the XUV Photometer System (XPS) instrument onboard SORCE, measuring the irradiance with several large passbands. The measurements are then processed with an algorithm using CHIANTI spectral models (Woods et al. 2008). A composite spectrum, with 1-nm bin resolution, is used, based on the observations of those three instruments. As those missions are Earth-located, a simple dilution law $\left(\frac{1}{r^{2}}\right)$ is used to estimate the flux at the Jovian system. In Section 6, different values for the solar UV fluxes will be used for inferring the sensibility of the respective upper atmospheric emissions. 

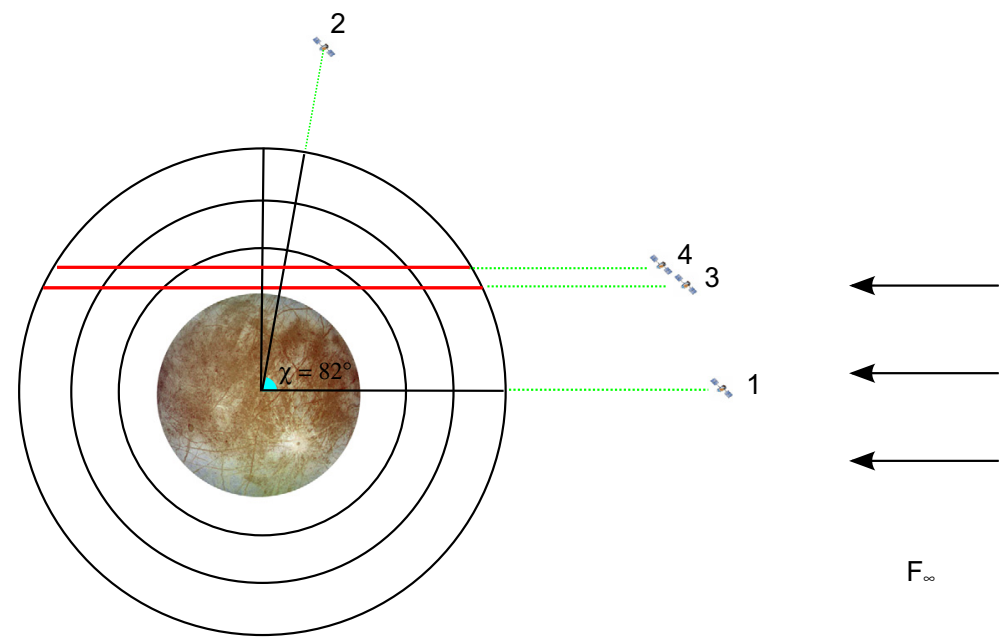

Fig. 2. Modeling geometries. Cases 1 and 2 correspond to a nadir view for both equatorial and polar regions at noon. Cases 3 and 4 correspond to a limb view, with the closest altitude to Europa's surface being, respectively, 10 and $100 \mathrm{~km}$. The red lines illustrate the line of sight within the atmosphere.

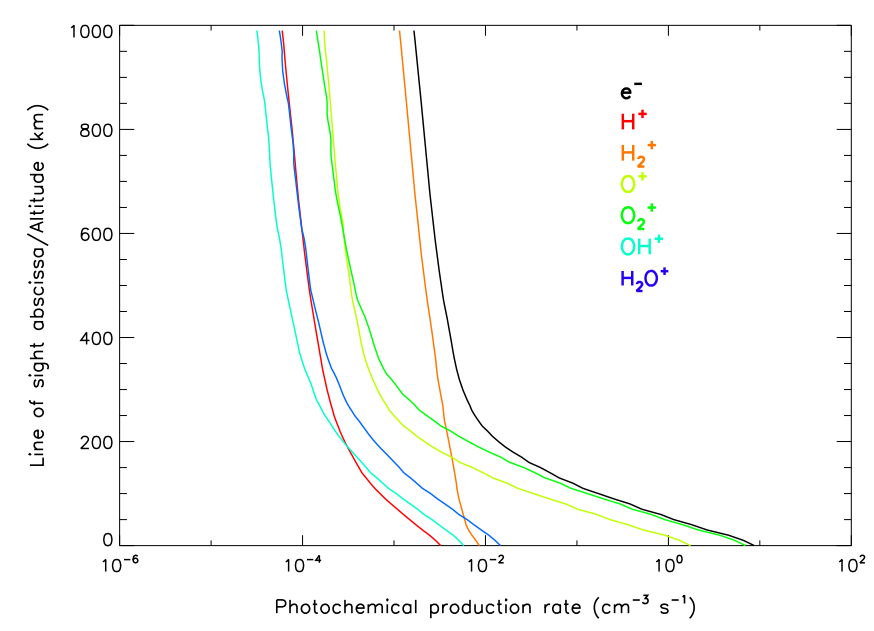

Fig. 3. Photochemical production rates for all ions species and the electron production rate for nadir viewing at the equator (case 1) for high solar activity.

For modeling the atmospheric response to the solar UV flux, a Beer-Lambert law is considered. The solar UV flux at each altitude $z, F(\lambda, z)$, is computed within the Europa's atmosphere, such as:

$$
F(\lambda, z)=F_{\infty}(\lambda) e^{-\tau_{\text {tot }}}
$$

where $F_{\infty}$ represents the flux at the top of the atmosphere, i.e. the incoming solar UV flux, and $\tau_{\text {tot }}$ represents the optical depth, computed from:

$$
\tau_{\text {tot }}=\sum_{k} \sigma_{\text {tot }}^{k}(\lambda) \int_{z}^{\infty} n_{k}\left(z^{\prime}\right) \mathrm{d} z^{\prime},
$$

where $\sigma_{\text {tot }}^{k}$ are the wavelength-dependent total photoabsorption cross-sections for the six species, indexed $k . n_{k}$ is the neutral density at altitude $z$ for each species $k$. These values are taken from the neutral atmospheric model.

The partial photoionization and photoexcitation crosssections $\left(\sigma_{a}^{k}\right)$ for several states (indexed $a$ ) are considered to calculate ion production rates (for $\mathrm{H}_{2} \mathrm{O}^{+}, \mathrm{OH}^{+}, \mathrm{O}_{2}^{+}, \mathrm{O}^{+}, \mathrm{H}_{2}^{+}$, and $\mathrm{H}^{+}$), the electron production rate and production rates

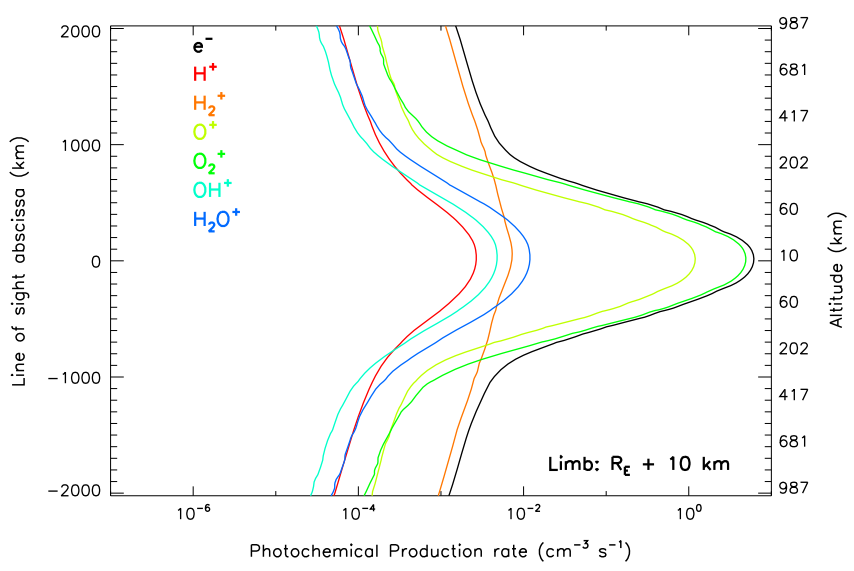

Fig. 4. Photochemical production rates for all ions species and the photo-electron production rate for limb viewing (case 3) for high solar activity. The left $y$-axis corresponds to the line of sight abscissa, and the right $y$-axis indicates the corresponding altitude.

for different atomic oxygen states (such as $\mathrm{O}\left({ }^{3} \mathrm{P}\right), \mathrm{O}\left({ }^{1} \mathrm{D}\right)$, and $\left.\mathrm{O}\left({ }^{1} \mathrm{~S}\right)\right)$. The production rate, $P_{k}^{a}$, is computed as follows:

$$
P_{k}^{a}(z)=\int_{\lambda} n_{k}(z) \sigma_{a}^{k}(\lambda) F(\lambda, z) \mathrm{d} \lambda .
$$

The total and partial photoabsorption cross-sections used throughout this study have been summarized by Cessateur et al. (2012a).

\subsection{Photoionization}

The results of the photoionization in case 1 for a high solar activity $(\mathrm{F} 10.7=195)$ are displayed in Figure 3 . Very similar profiles have been obtained for case 2 , with nadir observations at $82^{\circ}$, despite a different geometry. In both cases 1 and 2 , the maximum of ionization occurs at the surface, with an electron production rate of about $8.6 \mathrm{~cm}^{-3} \mathrm{~s}^{-1}$. The predominant photochemically produced ion in this region is $\mathrm{O}_{2}^{+}$, whereas at higher altitudes (above roughly $250 \mathrm{~km}$ ), it becomes $\mathrm{H}_{2}^{+}$.

Figure 4 displays the results of the photoionization in case 3, also for a high solar activity. The electron and ion productions are maximal where the atmosphere is the densest, 
Table 2. Thermal electron density (column density in $\mathrm{cm}^{-2}$ ) integrated along the line of sight using the atmospheric model 1 for the four different cases and for two different solar activities.

\begin{tabular}{lcc}
\hline \hline & $\begin{array}{c}\text { Solar activity } \\
(\mathrm{F} 10.7)\end{array}$ & $\begin{array}{c}\text { Total thermal electron density, } \\
\text { or TEC }\left(\mathrm{cm}^{-2}\right)\end{array}$ \\
\hline Case 1 & 83 & $5.29 \times 10^{10}$ \\
& 195 & $6.49 \times 10^{10}$ \\
\hline Case 2 & 83 & $5.24 \times 10^{10}$ \\
& 195 & $6.44 \times 10^{10}$ \\
\hline Case 3 & 83 & $4.89 \times 10^{11}$ \\
& 195 & $6.04 \times 10^{11}$ \\
\hline Case 4 & 83 & $1.63 \times 10^{11}$ \\
& 195 & $1.66 \times 10^{11}$ \\
\hline
\end{tabular}

i.e. at $10 \mathrm{~km}$ above the surface, with an electron production rate of about $6.1 \mathrm{~cm}^{-3} \mathrm{~s}^{-1}$. The profiles look symmetric because the extinction is very small since Europa's atmosphere is very thin. However, the electron production is different at the entrance (at $2022 \mathrm{~km}$ ) compared to the exit $(-2022 \mathrm{~km})$ by about $18 \%$. Similar profiles have been obtained for case 4 , but with significant lower photochemical production rates. At $100 \mathrm{~km}$ above the surface, the electron production rate reaches $0.17 \mathrm{~cm}^{-3} \mathrm{~s}^{-1}$.

From the electron production rate, the thermal electron population within Europa's atmosphere can be estimated. In order to retrieve the electron density from the production rate, a steady state has been assumed, where production mechanisms are equivalent to loss mechanisms. According to Figures 3 and 4, there are two dominant ions with $\mathrm{O}_{2}^{+}$and $\mathrm{H}_{2}^{+}$, regardless of the altitude. The following loss reactions $\mathrm{H}_{2}^{+}+e_{\mathrm{th}}^{-} \rightarrow 2 \mathrm{H}$, and $\mathrm{O}_{2}^{+}+e_{\mathrm{th}}^{-} \rightarrow 2 \mathrm{O}$ are taken into account, with coefficient rates of $2.3 \times 10^{-7}\left(300 / T_{e}\right)^{0.4} \mathrm{~cm}^{3} \mathrm{~s}^{-1}$ and $2 \times 10^{-7}\left(300 / T_{\mathrm{e}}\right)^{0.7} \mathrm{~cm}^{3} \mathrm{~s}^{-1}$, respectively (Florescu-Mitchell $\&$ Mitchell 2006). $T_{e}$ represents the temperature of thermalized electrons. These reactions are known as the principal means of ion recombination within ionospheres (Banks \& Kockarts 1973) and are more likely to occur than the simple recombination reaction (resulting in $\mathrm{H}_{2}$ and $\mathrm{O}_{2}$ ). The electron temperature is necessarily larger than or equal to the neutral temperature. As an approximation, the same temperature, as for $\mathrm{H}_{2}$ provided by Smyth \& Marconi (2006), of about $1000 \mathrm{~K}$ is considered. Table 2 summarizes the integrated electron densities (or column densities) along the line of sight, obtained with this rather simple chemical model. However, these values represent some lower limits, since neither electron precipitation, ion-neutral charge exchange, nor secondary photoionization within Europa's atmosphere has been considered. Some other loss processes should also be taken into account such as ion escape. However, Cessateur et al. (2012a) have applied the same model to estimate the electron density within Ganymede's atmosphere. The fact that a good agreement with in-situ measurements performed by Galileo (Gurnett et al. 1996) was found in that paper provides evidence for the validity of the applied methodological approach. The solar activity over the 11-year cycle leads to a total thermal electron density variability of $23 \%$ for cases $1-3$, while it is only $2 \%$ for case 4 .

\subsection{Photodissociation and photoexcitation}

Excitation production rates for several states of the atomic oxygen are now computed, namely the $\mathrm{O}\left({ }^{3} \mathrm{P}\right), \mathrm{O}\left({ }^{1} \mathrm{D}\right)$, and $\mathrm{O}\left({ }^{1} \mathrm{~S}\right)$, in
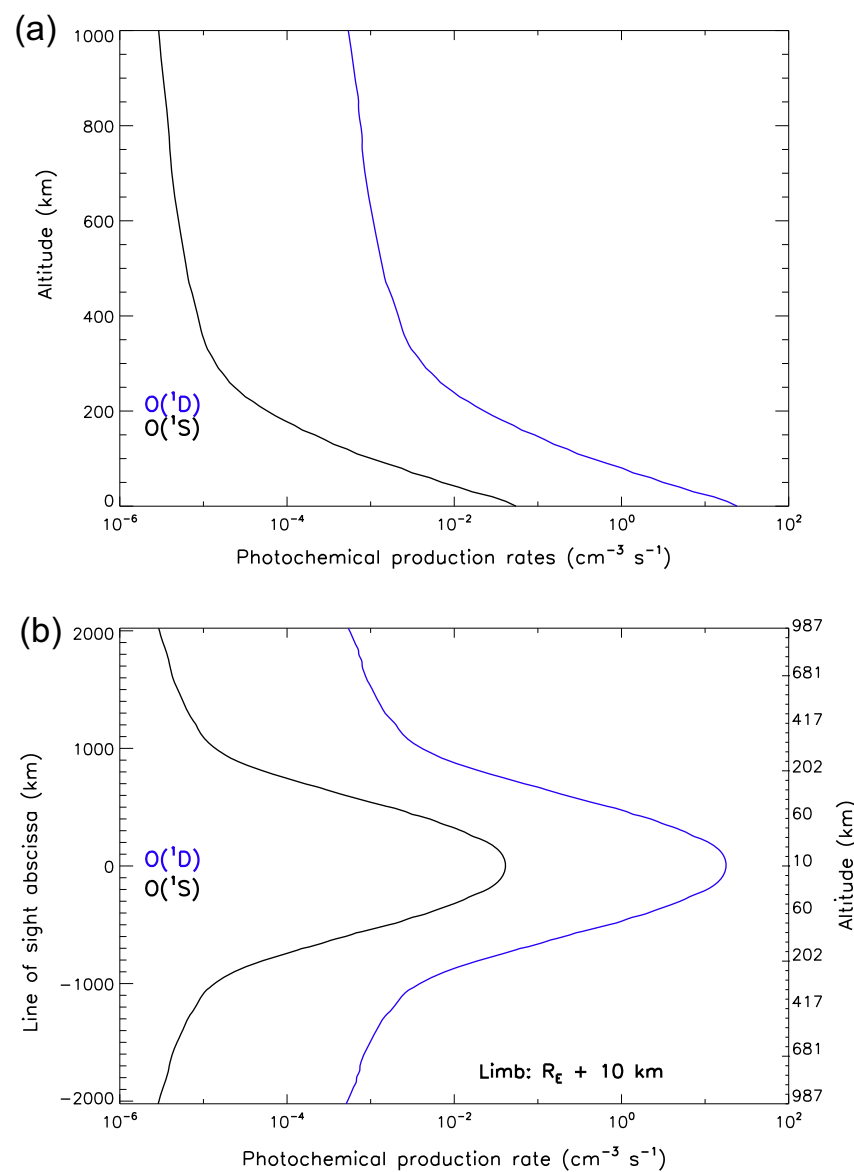

Fig. 5. Photochemical production rates of $O\left({ }^{1} D\right)$ and $O\left({ }^{1} S\right)$ from neutral species for equatorial viewing in case 1 (a) and limb viewing in case 3 (b), for high solar activity.

the same way as for the photoionization. Those results are displayed for cases 1 and 3 in Figure 5, for high solar activity. Profiles for case 2 are very similar to case 1 . In case 4 , similar profiles to case 3 have been calculated but with significant lower production rates. While the $\mathrm{O}\left({ }^{3} \mathrm{P}\right)$ production rate maximum is $32 \mathrm{~cm}^{-3} \mathrm{~s}^{-1}$ for case 3 , it is only $0.8 \mathrm{~cm}^{-3} \mathrm{~s}^{-1}$ for case 4. As for photoionization processes, atomic oxygen production rates maximize where the atmosphere is densest, i.e. close to the ground. For case 1, our model suggests a ratio of about 0.20 and $1.2 \times 10^{-3}$ for $\mathrm{O}\left({ }^{1} \mathrm{D}\right) / \mathrm{O}\left({ }^{3} \mathrm{P}\right)$ and $\mathrm{O}\left({ }^{1} \mathrm{~S}\right) / \mathrm{O}\left({ }^{3} \mathrm{P}\right)$, respectively. Similar values have been obtained for all other cases.

\section{Dayglow emission modeling}

From these previous results, estimation of the dayglow emission for several spectral lines can be made. Model 1 (i.e. Smyth \& Marconi 2006) is first used for estimating dayglow emission. Comparisons between atmospheric models will be performed in Section 3.3. Two line emissions are of interest here: the radiative transition from $\mathrm{O}\left({ }^{1} \mathrm{D}\right)$ to $\mathrm{O}\left({ }^{3} \mathrm{P}\right)$, which is responsible for the formation of the red line emission at $630 \mathrm{~nm}$ and the deactivation of the $\mathrm{O}\left({ }^{1} \mathrm{~S}\right)$ state leading to the green line emission at $557.7 \mathrm{~nm}$. Both emission measurements are of primary importance since the green to red lines ratio is commonly used for determining the abundances of $\mathrm{CO}$ and $\mathrm{CO}_{2}$ relative to the $\mathrm{H}_{2} \mathrm{O}$ abundance in planetary and cometary atmospheres. Cessateur et al. (2012a) have shown that emission from the deactivation of $\mathrm{OH}\left(\mathrm{A}^{2} \Sigma^{+}\right)$to $\mathrm{OH}\left(\mathrm{X}^{2} \Pi\right)$ is very small in the 
Table 3. Production and loss reactions of $\mathrm{O}\left({ }^{1} \mathrm{D}\right)$ and $\mathrm{O}\left({ }^{1} \mathrm{~S}\right)$.

\begin{tabular}{lll}
\hline \hline Reaction & \multicolumn{1}{c}{ Rate $^{\mathrm{a}}$} & Reference \\
\hline$\left(\mathrm{H}_{2} \mathrm{O}, \mathrm{OH}, \mathrm{O}_{2}, \mathrm{O}\right)+h v \rightarrow\left(\mathrm{O}\left({ }^{1} \mathrm{D}\right), \mathrm{O}\left({ }^{1} \mathrm{~S}\right)\right)$ & Model & This work \\
$\mathrm{O}_{2}^{+}+e_{\mathrm{th}}^{-} \rightarrow\left(\mathrm{O}\left({ }^{1} \mathrm{D}\right), \mathrm{O}\left({ }^{1} \mathrm{~S}\right)\right)+\mathrm{O}\left({ }^{3} \mathrm{P}\right)$ & $2 \times 10^{-7}\left(300 / T_{\mathrm{e}}\right)^{0.7}$ & Florescu-Mitchell \& Mitchell $(2006)$ \\
\hline$(1) \mathrm{O}\left({ }^{1} \mathrm{D}\right)+\mathrm{O}_{2} \rightarrow \mathrm{O}\left({ }^{3} \mathrm{P}\right)+\mathrm{O}_{2}$ & $k_{1}=3.2 \times 10^{-11}$ & Atkinson et al. $(2004)$ \\
$(2) \mathrm{O}\left({ }^{1} \mathrm{D}\right)+\mathrm{O} \rightarrow \mathrm{O}\left({ }^{3} \mathrm{P}\right)+\mathrm{O}\left({ }^{3} \mathrm{P}\right)$ & $k_{2}=2.5 \times 10^{-11}$ & Kalogerakis et al. $(2009)$ \\
$(3) \mathrm{O}\left({ }^{1} \mathrm{D}\right)+e_{\mathrm{th}}^{-} \rightarrow \mathrm{O}\left({ }^{3} \mathrm{P}\right)+e_{\mathrm{th}}^{-}$ & $k_{3}=8.3 \times 10^{-10}$ & Pavlov et al. (1999) \\
$(4) \mathrm{O}\left({ }^{1} \mathrm{D}\right)+\mathrm{H}_{2} \mathrm{O} \rightarrow \mathrm{OH}+\mathrm{OH}$ & $k_{4}=2.2 \times 10^{-10}$ & Schmidt et al. $(1988)$ \\
$(5) \mathrm{O}\left({ }^{1} \mathrm{D}\right) \rightarrow \mathrm{O}\left({ }^{3} \mathrm{P}\right)+h v(630 \mathrm{~nm})$ & $A_{1}=6.478 \times 10^{-3}$ & Fischer \& Tachiev $(2004)$ \\
$(6) \mathrm{O}\left({ }^{1} \mathrm{D}\right) \rightarrow \mathrm{O}\left({ }^{3} \mathrm{P}\right)+h v(634.4 \mathrm{~nm})$ & $A_{2}=2.097 \times 10^{-3}$ & Fischer \& Tachiev $(2004)$ \\
$(7) \mathrm{O}\left({ }^{1} \mathrm{~S}\right) \rightarrow \mathrm{O}\left({ }^{1} \mathrm{D}\right)+h v(557.7 \mathrm{~nm})$ & $A_{3}=1.26$ & Wiese et al. $(1996)$ \\
$(8) \mathrm{O}\left({ }^{1} \mathrm{~S}\right) \rightarrow \mathrm{O}\left({ }^{3} \mathrm{P}\right)+h v(297.7 \mathrm{~nm})$ & $A_{4}=0.134$ & Slanger et al. $(2006)$ \\
$(9) \mathrm{O}\left({ }^{1} \mathrm{~S}\right)+\mathrm{O}_{2} \rightarrow \mathrm{O}\left({ }^{3} \mathrm{P}\right)+\mathrm{O}_{2}$ & $k_{5}=3.2 \times 10^{-13}$ & Stuhl \& Welge $(1969)$ \\
$(10) \mathrm{O}\left({ }^{1} \mathrm{~S}\right)+\mathrm{H}_{2} \mathrm{O} \rightarrow \mathrm{OH}+\mathrm{OH}$ & $k_{6}=3 \times 10^{-10}$ & Zipf $(1969)$ \\
\hline
\end{tabular}

${ }^{\text {a }}$ Units are $\mathrm{cm}^{3} \mathrm{~s}^{-1}$ for two-body reactions, $\mathrm{s}^{-1}$ for photolysis reactions and $A_{i}$ are in $\mathrm{s}^{-1}$.

case of Ganymede. Since the $\mathrm{H}_{2} \mathrm{O}$ and $\mathrm{O}_{2}$ vertical density profiles are of the same order of magnitude within Europa's atmosphere, these emissions will not be computed here.

\section{1. $O\left({ }^{1} D\right)$ and $O\left({ }^{1} S\right)$ emission lines}

In order to compute aeronomic emissions, Cessateur et al. (2012a) assumed that radiative transition was the most effective way to deactivate excited species. Therefore different other loss mechanisms have been neglected. Several possible production and loss mechanisms for $\mathrm{O}\left({ }^{1} \mathrm{D}\right)$ and $\mathrm{O}\left({ }^{1} \mathrm{~S}\right)$ have been identified by many studies (see e.g. Thirupathaiah \& Singh 2014). In this current paper, significant chemical reactions are considered in order to provide accurate dayglow emission estimates. For the case of Europa's atmosphere, the different loss mechanisms as well as the production mechanisms are summarized in Table 3. The values of the rate constants and transition probabilities are also listed.

\subsubsection{Production mechanisms of $O\left({ }^{1} D\right)$ and $O\left({ }^{l} S\right)$}

The photoexcitation of the water group species $\left(P_{P E}\right)$ is an important production pathway of the atomic $O\left({ }^{1} \mathrm{D}\right)$ and $\mathrm{O}\left({ }^{1} \mathrm{~S}\right)$ states. Figure 5 provides the radial profile of the total production rates for $\mathrm{O}\left({ }^{1} \mathrm{D}\right)$ and $\mathrm{O}\left({ }^{1} \mathrm{~S}\right)$. The production rates here only include the impact of the solar UV flux on the water group species excluding other known processes. The dissociative recombination, $\left(P_{D R}\right)$, of $\mathrm{O}_{2}^{+}$is also considered, i.e.,

$$
\mathrm{O}_{2}^{+}+e_{\mathrm{th}}^{-} \rightarrow \mathrm{O}^{*}+\mathrm{O}\left({ }^{3} \mathrm{P}\right),
$$

where $\mathrm{O}^{*}=\mathrm{O}\left({ }^{1} \mathrm{D}\right), \mathrm{O}\left({ }^{1} \mathrm{~S}\right)$, and $\mathrm{O}\left({ }^{5} \mathrm{~S}\right)$. This reaction is generally an important source of atomic oxygen emissions (Banks \& Kockarts 1973), and in particular for Europa (Nagy et al. 1998). The electrons involved in this reaction are mostly thermalized. As an approximation, a steady state is considered, where production and loss terms for the $\mathrm{O}_{2}^{+}$ion are supposed to be equal. Production rates of $\mathrm{O}_{2}^{+}$for several orbital configurations have been previously computed (see Figs. 3 and 4). The branching ratio for inferring the proportion of $\mathrm{O}\left({ }^{1} \mathrm{D}\right), \mathrm{O}\left({ }^{1} \mathrm{~S}\right)$, and $\mathrm{O}\left({ }^{3} \mathrm{P}\right)$ resulting from the dissociative recombination is provided by Kella et al. (1997). This allows us to derive the atmospheric emissions. Dissociative recombination of $\mathrm{H}_{2} \mathrm{O}^{+}$is neglected in the case of Europa, since the global water concentration considered here with Models 1 and 2 is particularly low. However, the existence of plumes in Europa as recently observed with HST could locally increase the $\mathrm{H}_{2} \mathrm{O}$ and $\mathrm{H}_{2} \mathrm{O}^{+}$concentrations (Roth et al. 2014). The deactivation of $\mathrm{O}\left({ }^{1} \mathrm{~S}\right)$ by radiative decay at $557.7 \mathrm{~nm}$ leads also to the production of the $\mathrm{O}\left({ }^{1} \mathrm{D}\right)$ state (see reaction (7)).

\subsubsection{Loss mechanisms of $O\left({ }^{l} D\right)$ and $O\left({ }^{l} S\right)$}

Several significant chemical reactions and radiative transitions are taken into account as loss mechanisms of $\mathrm{O}\left({ }^{1} \mathrm{D}\right)$ and $\mathrm{O}\left({ }^{1} \mathrm{~S}\right)$, listed as reactions (1)-(10) in Table 3. For reactions (1), (2), and (4), the concentration profiles for $\mathrm{O}_{2}, \mathrm{O}$, and $\mathrm{H}_{2} \mathrm{O}$ are taken from the Europa's atmospheric model (see Fig. 1). The thermal electronic density for reaction (3) is deduced from the thermal electronic production rate computed in the previous Section 2.3. Figure 6 displays the different loss rates for the $\mathrm{O}\left({ }^{1} \mathrm{D}\right)$ state, for cases 3 and 4 . Starting with case 3 , the collisional quenching of the excited oxygen atoms by $\mathrm{O}_{2}$ is a very effective loss process for altitudes lower than $80 \mathrm{~km}$. The loss rate reaches $5.6 \times 10^{-3} \mathrm{~s}^{-1}$ close to the ground. The collisional quenching by $\mathrm{H}_{2} \mathrm{O}$ shall also be considered but plays a minor role here with a loss rate of about $1.6 \times 10^{-4} \mathrm{~s}^{-1}$ close to the ground. Regardless of the neutral and electronic temperatures, reactions (2) and (3) are not efficient for quenching the produced $\mathrm{O}\left({ }^{1} \mathrm{D}\right)$ with maximal loss rate values of about $1.52 \times 10^{-6} \mathrm{~s}^{-1}$ and $5.2 \times 10^{-6} \mathrm{~s}^{-1}$, respectively. Above $80 \mathrm{~km}$, loss processes are mainly dominated by radiative processes leading to the red line emission at $630 \mathrm{~nm}$. For case 4, with limb viewing $100 \mathrm{~km}$ above the surface, the $\mathrm{O}_{2}$ concentration is not high enough in order to allow collisional quenching to be an effective loss process. Along the line of sight for case 4 , deactivation of the $O\left({ }^{1} D\right)$ goes through the radiative process with red line emission.

\subsection{Red and green line dayglow emission estimations}

In order to infer the red and green line emissions, the number densities of $\mathrm{O}\left({ }^{1} \mathrm{D}\right)$ and $\mathrm{O}\left({ }^{1} \mathrm{~S}\right)$ within Europa's atmosphere have first to be computed. To do so, we solve the continuity equation for steady-state equations and spherical symmetry, such as

$$
\frac{1}{r^{2}} \frac{d}{d r}\left(r^{2} N_{i} v_{i}\right)=P_{i}-L_{i}
$$

where $N_{i}$ are the number densities for the excited atomic oxygen states, i.e. $\mathrm{O}\left({ }^{1} \mathrm{D}\right)$ and $\mathrm{O}\left({ }^{1} \mathrm{~S}\right) . P_{i}$ and $L_{i}$ are the production and loss rates, respectively, as described in the previous sections. The radial velocity profiles, $v_{i}$, for neutral oxygen 
G. Cessateur et al.: Photochemistry-emission coupled model for Europa and Ganymede
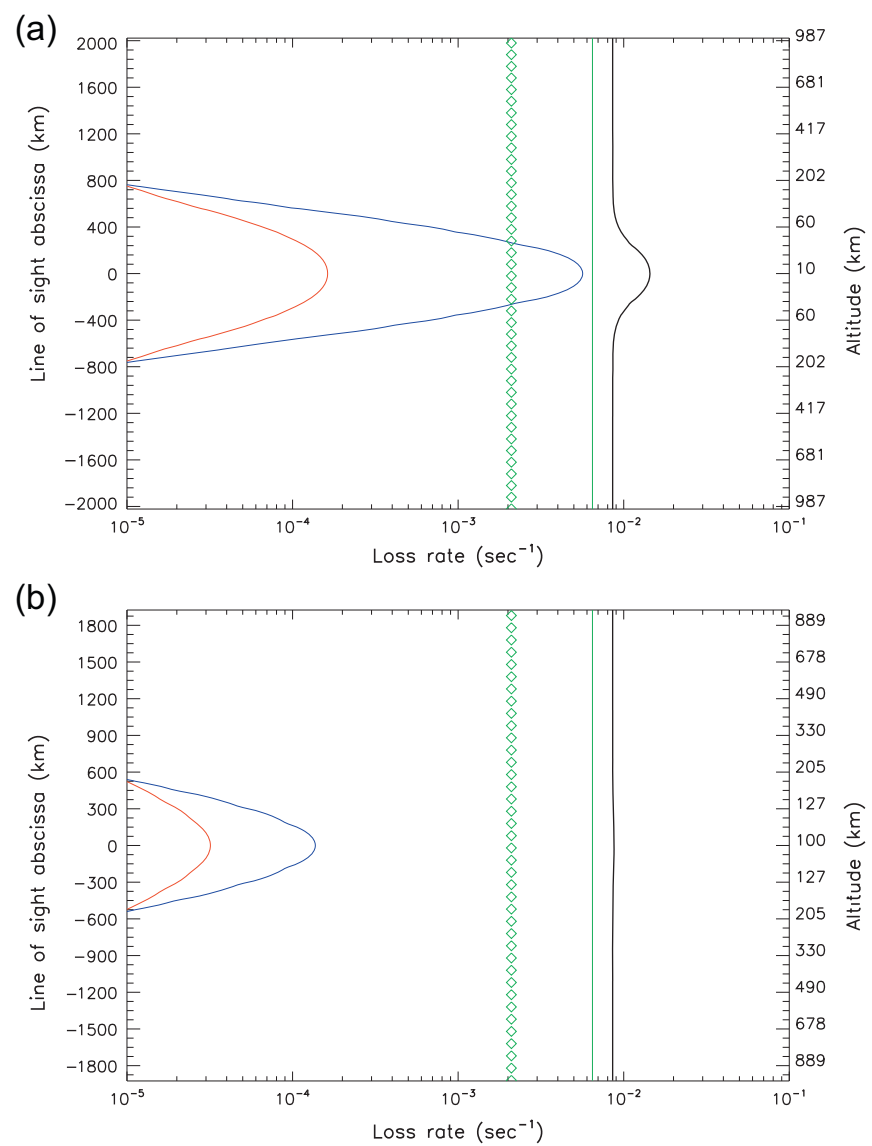

Fig. 6. Total loss rate for $O\left({ }^{1} \mathrm{D}\right)$ for limb viewing at $10 \mathrm{~km}(\mathrm{a})$ and $100 \mathrm{~km}$ (b) above Europa's surface in black. Partial loss rates for radiative decay (in green thin line for reaction (5), in green dotted line for reaction (6)), for collisions with $\mathrm{O}_{2}$ (in blue), and for collisions with $\mathrm{H}_{2} \mathrm{O}$ (in red) are also indicated.

atoms have been taken from Shematovich et al. (2005): below roughly $23 \mathrm{~km}$, velocities are negative, i.e. the oxygen atoms are precipitating toward the surface, with velocities of around $100 \mathrm{~m} \mathrm{~s}^{-1}$ at $10 \mathrm{~km}$ above the surface. For altitudes above $23 \mathrm{~km}$, velocities are positive, i.e. the oxygen atoms are escaping. The transport will not impact the $O\left({ }^{1} \mathrm{~S}\right)$ density because its lifetime is only $0.8 \mathrm{~s}$. Oxygen atoms in the $\mathrm{O}\left({ }^{1} \mathrm{D}\right)$ state with a lifetime of about $130 \mathrm{~s}$, which represents a distance of $10-15 \mathrm{~km}$ at the considered velocity, will however be impacted. The computed densities are displayed in Figure 7 for case 2 (nadir viewing at $82^{\circ}$ ). For the sake of comparison, the number density of $\mathrm{O}\left({ }^{1} \mathrm{D}\right)$ without transport is also represented.

The difference arising from the inclusion of the transport is significant through the whole line of sight. The $O\left({ }^{1} \mathrm{D}\right)$ number density is divided by 2 for altitudes lower than $15 \mathrm{~km}$. Indeed, because of a high velocity, a large proportion of oxygen atoms in the $O\left({ }^{1} D\right)$ state will impact the surface before radiating at $630 \mathrm{~nm}$, which causes this depletion. As a boundary value, the $O\left({ }^{1} \mathrm{D}\right)$ number density is set to 0 at the surface. For altitudes above $100 \mathrm{~km}$, where neutral oxygen atoms velocities are positive, the number density is shifted toward higher altitudes. Around $23 \mathrm{~km}$, the transition zone occurs where the oxygen atoms have a mean velocity close to 0 which explains the local increase of the density with values close to photochemical equilibrium conditions (i.e. without transport). Generally, taking the transport into account leads to an

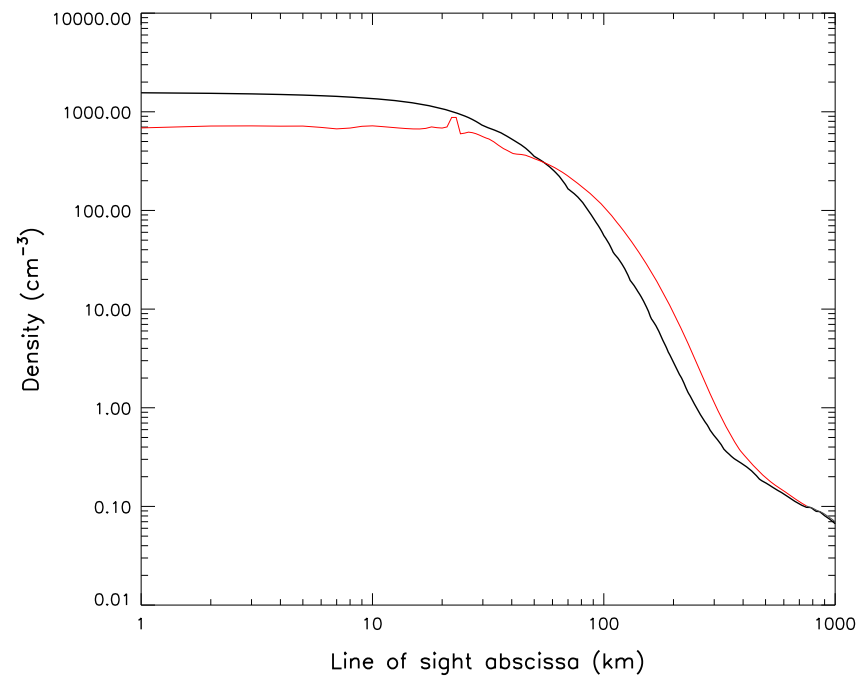

Fig. 7. $O\left({ }^{1} \mathrm{D}\right)$ number densities from photo impact-induced excitation, with (red line) and without transport (black line) for case 2.

Table 4. Red and green line emissions in Rayleigh from photo impact-induced excitation, using the atmospheric model 1 for the four different cases and for two different solar activities.

\begin{tabular}{cccc}
\hline \hline & $\begin{array}{c}\text { Solar } \\
(\mathrm{F} 10.7)\end{array}$ & $\begin{array}{c}\text { Activity } \mathrm{O}\left({ }^{1} \mathrm{D}-{ }^{3} \mathrm{P}\right) \\
\text { (redline) }\end{array}$ & $\begin{array}{c}\mathrm{O}\left({ }^{1} \mathrm{~S}-{ }^{1} \mathrm{D}\right) \\
\text { (greenline) }\end{array}$ \\
\hline Case 1 & 83 & 28.5 & 0.41 \\
& 195 & 34.6 & 0.62 \\
\hline Case 2 & 83 & 28.3 & 0.41 \\
& 195 & 34.2 & 0.61 \\
\hline Case 3 & 83 & 338.3 & 5.45 \\
& 195 & 408.5 & 8 \\
\hline Case 4 & 83 & 46 & 0.3 \\
& 195 & 55.6 & 0.32 \\
\hline
\end{tabular}

$\mathrm{O}\left({ }^{1} \mathrm{D}\right)$ depletion for altitudes below $50 \mathrm{~km}$, and an $\mathrm{O}\left({ }^{1} \mathrm{D}\right)$ enrichment for higher altitudes.

The red and green line emissions are directly deduced from the number densities by multiplying $N_{i}$ by the corresponding Einstein transition probability, i.e. $A_{1}$ and $A_{3}$ for the red line at $630 \mathrm{~nm}$ and the green line at $557 \mathrm{~nm}$, respectively. The results for Europa's atmospheric emissions are summarized for the different orbital configurations and for different solar activities in Table 4. Similar values have been obtained for both nadir cases from $28 \mathrm{R}$ to $34 \mathrm{R}$ according to the solar activity. Let us precise that taking the transport into account leads to an emission decrease of about $23 \%$ for nadir cases because of the local depletion at lower altitudes and the loss by impact with the surface. For the limb viewing case 3, values are considerably higher compared to cases 1 and 2 only because of the longer line of sight within Europa's atmosphere, from $338.3 \mathrm{R}$ to $408.5 \mathrm{R}$ according to the solar activity. For case 3 , taking the transport into account leads to an emission decrease of about $30.8 \%$. For case 4 , at $100 \mathrm{~km}$ above the surface, the emissions vary from $46 \mathrm{R}$ to $55.6 \mathrm{R}$ according to the solar activity. Red line emissions in case 4 are increased by a factor of about $125 \%$ compared to calculations without transport, which is explained by the $\mathrm{O}\left({ }^{1} \mathrm{D}\right)$ enrichment at high altitudes.

The Jovian system is undoubtedly affected by the solar activity at any time scale (see Jackman \& Arridge 2011). 


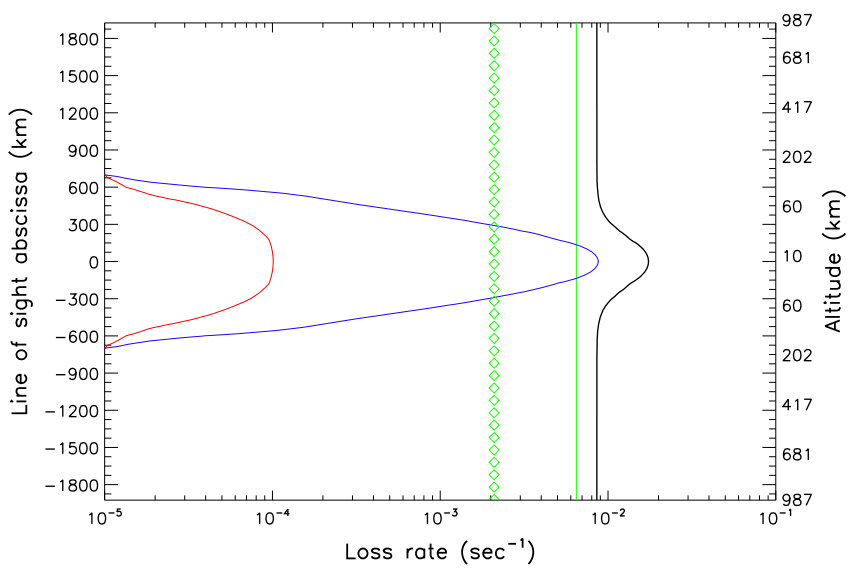

Fig. 8. Total loss rate for $\mathrm{O}\left({ }^{1} \mathrm{D}\right)$ for limb viewing $10 \mathrm{~km}$ above Europa's surface in black, using the atmospheric model 2. Partial loss rates for radiative decay (in green thin line for reaction (5), in green dotted line for reaction (6)), for collisions with $\mathrm{O}_{2}$ (in blue) and for collisions with $\mathrm{H}_{2} \mathrm{O}$ (in red) are also depicted.

Dayglow emissions are also impacted by the solar activity, but the effect is not linear. Both quiet and active solar activity conditions have been tested with F10.7 equal to 83 and 195, respectively. Dayglow emissions are increased by about $21 \%$ for every case considered here in this study. Actual spectrometers are nowadays sensitive enough to be able to detect such emissions for limb viewing (i.e. cases 3 and 4). Nadir cases are however not suitable for observations because of the reflected solar illumination on the surface. Therefore relative density measurements can be skewed by up to $21 \%$ if solar activity is neglected.

The green line emission at $577.7 \mathrm{~nm}$ is estimated in the same way. The radiative decay is very high $\left(A_{3}=1.26 \mathrm{~s}^{-1}\right)$ compared to the loss rate resulting from the collisional reactions $\left(7.4 \times 10^{-5} \mathrm{~s}^{-1}\right.$ and $2.72 \times 10^{-7} \mathrm{~s}^{-1}$ for reactions (9) and (10), respectively, at the ground). In the case of Europa's atmosphere, radiative decay is the most effective way to deactivate the $\mathrm{O}\left({ }^{1} \mathrm{~S}\right)$ state of the atomic oxygen, with a quenching ratio of 0.9 . The emissions by photo impact-induced excitation only, summarized in Table 4, are small, with a maximum of $8 \mathrm{R}$ in case of the limb viewing (case 3) for a high solar activity. For case 4 , the green line is pretty weak, close to $0.3 \mathrm{R}$, which might be difficult to detect. The green line emissions from photo impact-induced excitation are weak and will be discarded in the following.

\subsection{Comparison with other Europa atmospheric models}

Observations of dayglow emissions represent valuable information in order to constrain atmospheric species densities for planetary atmospheres. Dayglow emissions for the red and green lines have been estimated using the neutral atmospheric Model 1 (i.e. from Smyth \& Marconi 2006). We consider from now on Model 2. The concentration profiles of $\mathrm{O}_{2}, \mathrm{O}, \mathrm{H}_{2} \mathrm{O}$, and $\mathrm{OH}$ have been displayed in Figure 1. As a reminder, there is a ratio of about 1.5 and 4 for column densities of $\mathrm{O}_{2}$ and $\mathrm{O}$, respectively, between the two neutral atmospheric models. Since $\mathrm{H}_{2}$ and $\mathrm{H}$ are not provided, the previous density profiles from Model 1 are used. This will not affect our conclusions, since these two species, especially $\mathrm{H}_{2}$, are more important for the photoionization processes.

The loss rate using the atmospheric Model 2 is displayed in Figure 8 . The loss rate is dominated by collisional reaction
Table 5. Red line emissions in Rayleigh using the model of Shematovich et al. (2005). Differences with the model of Smyth \& Marconi (2006) in brackets.

\begin{tabular}{lcc}
\hline \hline & Solar $(\mathrm{F} 10.7)$ & Activity $\mathrm{O}\left({ }^{1} \mathrm{D}-{ }^{3} \mathrm{P}\right)($ redline $)$ \\
\hline Case 1 & 83 & $34.3(+20.3 \%)$ \\
& 195 & $41.5(+19.9 \%)$ \\
\hline Case 3 & 83 & $385.9(+13.9 \%)$ \\
& 195 & $465(+14 \%)$ \\
\hline \multirow{2}{*}{ Case 4} & 83 & $35.9(-21.9 \%)$ \\
& 195 & $43.5(-23.2 \%)$ \\
\hline
\end{tabular}

with $\mathrm{O}_{2}$ for altitudes lower than $30 \mathrm{~km}$, and the maximal loss rate is $1.7 \times 10^{-2} \mathrm{~s}^{-1}$ at $10 \mathrm{~km}$ above Europa's surface. Above $30 \mathrm{~km}$, radiative decay is the dominant loss reaction. Table 5 summarizes the red line emission obtained with the model of Shematovich et al. (2005). Despite a higher loss rate, a 14\% increase of the red line emission is estimated for case 3. For observations at the equator, estimations have been increased of $20 \%$. Red line emissions are not linearly dependent on the $\mathrm{O}_{2}$ concentration, because of the loss processes involving $\mathrm{O}_{2}$. However, an estimated $22-23 \%$ decrease of the red line emission is expected for case 4, for the limb viewing $100 \mathrm{~km}$ above Europa's surface. This result has to be related to the distribution along the altitude in $\mathrm{O}_{2}$ for both atmospheric models (Fig. 1).

The difference in terms of aeronomic emission $(+14 \%$ and $-22 \%$ for cases 3 and 4 , respectively) might be important enough to infer an accurate estimation of the $\mathrm{O}_{2}$ and $\mathrm{O}$ concentrations within Europa's atmosphere, providing a good knowledge of the solar UV flux, of the cross-section data, and of the relative brightness and morphologically distinguishable measurements for disentangling electron-impact excited emissions. Limb observations are clearly the best option for doing so, since dayglow emission estimations are high enough to be detected by actual onboard spectrometers. The signal will also not be polluted by the solar reflection from the surface. However, as will be explained in Section 6, these results are strongly dependent on the accuracy of the solar UV flux.

\section{Ganymede's dayglow emission}

Since the Moons and Jupiter Imaging Spectrometer (MAJIS) onboard JUICE will be devoted to the observations of both Europa and Ganymede, proper comparisons should be performed (Grasset et al. 2013). As previously mentioned, Cessateur et al. (2012a) already provided dayglow emission for the red and green lines in the case of Ganymede, but only for moderate solar activity $(\mathrm{F} 10.7=82)$. However, neither the collisional loss nor the transport of neutral oxygen has been taken into account. Indeed, radiative decay was the only loss mechanism considered. In the light of the presented model for Europa, $O\left({ }^{1} \mathrm{D}\right)$ loss through collision and transport cannot be neglected, and we provide here more realistic emission estimates for red line emissions from photo impact-induced excitation.

For the sake of comparison, the same orbital configurations as for Europa are considered. Ganymede has, however, a different neutral atmosphere as modeled by numerous studies (Marconi 2007; Turc et al. 2014; Plainaki et al. 2015) with two distinguished regions: a polar one with an $\mathrm{O}_{2^{-}}$and $\mathrm{H}_{2}$-dominated atmosphere, and an equatorial one where $\mathrm{H}_{2} \mathrm{O}$ 
G. Cessateur et al.: Photochemistry-emission coupled model for Europa and Ganymede

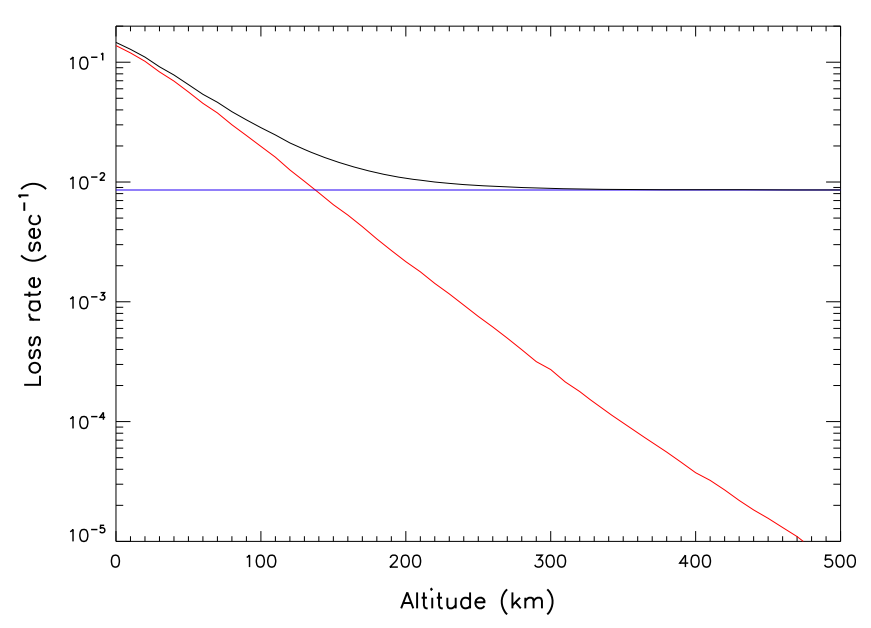

Fig. 9. Total loss rate for $\mathrm{O}\left({ }^{1} \mathrm{D}\right)$ for the equator region of Ganymede. Partial loss rates for radiative decay (in blue) and for collision with $\mathrm{H}_{2} \mathrm{O}$ (in red) are also illustrated.

and $\mathrm{H}_{2}$ are the most predominant species. The same reactions as listed in Table 3 for the production and loss mechanisms for the polar region are considered. The same reactions can also be applied to the equatorial region, but the dissociative recombination of $\mathrm{H}_{2} \mathrm{O}^{+}\left(\mathrm{H}_{2} \mathrm{O}^{+}+e_{\mathrm{th}}^{-} \rightarrow 2 \mathrm{H}+\mathrm{O}\right)$ must be added. A branching ratio of 0.35 for producing the $\mathrm{O}\left({ }^{1} \mathrm{D}\right)$ state from the dissociative recombination has been measured by Rosen et al. (2000). To take the transport into account, the mean radial velocity for oxygen atoms as computed by Marconi (2007) is considered. For polar regions at Ganymede, the radial velocity profile is very similar to the one at Europa. For equatorial regions, however, oxygen atoms always have a negative velocity, meaning that they are precipitating toward the surface. While the velocity is rather small (about $10 \mathrm{~m} \mathrm{~s}^{-1}$ ) at low altitudes, it reaches $900 \mathrm{~m} \mathrm{~s}^{-1}$ above $400 \mathrm{~km}$ up to $1000 \mathrm{~km}$.

The loss rates for Ganymede's polar region are very similar to the ones obtained for Europa (see Fig. 6). However, they are different for the equatorial region since $\mathrm{H}_{2} \mathrm{O}$ is the most predominant species there. Collisional quenching of the $\mathrm{O}\left({ }^{1} \mathrm{D}\right)$ state by $\mathrm{H}_{2} \mathrm{O}$ is the predominant loss reaction, especially for altitudes lower than $150 \mathrm{~km}$, with a loss rate of about $0.2 \mathrm{~s}^{-1}$ at the ground. For higher altitudes, the radiative decay of $\mathrm{O}\left({ }^{1} \mathrm{D}\right)$ is the most dominant loss process, as displayed in Figure 9.

Red line emission can be estimated for several orbital configurations as summarized in Table 6. A direct comparison with previous estimations from Cessateur et al. (2012a) is not realistic since collisional quenching and transport were not taken into account. For equatorial observations, red line emissions are estimated from $5.3 \mathrm{R}$ to $7.1 \mathrm{R}$ when taking the transport into account for the two solar activities which represents an emission increase of $40 \%$, between moderate and high solar activities. Photoexcitation of the $\mathrm{H}_{2} \mathrm{O}$ is dominated by the solar Lyman $\alpha$ line, with roughly $70 \%$ of the produced $O\left({ }^{1} \mathrm{D}\right)$ state. The solar UV flux at this particular wavelength should be known with great accuracy in order to constrain $\mathrm{H}_{2} \mathrm{O}$ density profiles within Ganymede's atmosphere at the equator when derived from visible dayglow data. Values for the nadir configuration at high latitudes are higher, from $20.6 \mathrm{R}$ to $25 \mathrm{R}$ depending on the solar activity which induced a variability of about $20 \%$. For a spacecraft looking at the limb, at $10 \mathrm{~km}$ above Ganymede's surface, red line emissions from $346.8 \mathrm{R}$
Table 6. Red line emission for Ganymede for cases 1-3 (in Rayleigh).

\begin{tabular}{lcc}
\hline \hline & Solar activity (F10.7) & This work \\
\hline Case 1 & 83 & 5.3 \\
& 195 & 7.1 \\
\hline Case 2 & 83 & 20.6 \\
& 195 & 25 \\
\hline Case 3 & 83 & 346.8 \\
& 195 & 418.7 \\
\hline
\end{tabular}

to $418.7 \mathrm{R}$ are estimated for solar UV photon impact-induced excitation only, according to the solar activity.

\section{Limitation of our approach}

Estimation of the red line emissions for Europa and Ganymede constitutes in fact a degenerate case study. Indeed, additionally to the solar UV flux impact, electron precipitation is expected to play an important role in the production of the $\mathrm{O}\left({ }^{1} \mathrm{D}\right)$ oxygen state. All the previous results are obtained without considering this additional source and could be considered as accurate in regions with no electron precipitations. As discussed previously, the relative importance of photochemical processes compared to electronic impact processes in the visible dayglow emissions has never been estimated in the cases of Europa and Ganymede. Electron impact, however, is considered to be the dominant dissociation mechanism and must be taken into account for assessing the global red line emission estimate for those moons. In order to assess the contribution of the photo-induced emissions, we consider here the auroral regions of Ganymede, which are then the most sensitive to electron impact.

As for the impact of the solar UV flux, we consider an electron distribution (in energy) convolved with the appropriate electron impact cross-section, as provided by the ATMOCIAD database (Gronoff et al. 2012). A Beer-Lambert law has been used (see Eqs. (1)-(3) in Sect. 2.2). This gives directly an estimate of the reaction rate associated to the electron impact reactions on $\mathrm{O}_{2}$, for a considered altitude in the atmosphere of the moon. The resulting red line emission is integrated along the line of sight. We consider here only case 2 for providing some estimates in the case of auroral regions. Based on Galileo data, we considered the electron distribution numerically described from $100 \mathrm{eV}$ to few $\mathrm{keV}$ (see Eq. (2) from Paranicas et al. 1999) in the case of Ganymede. The cross-section for the $\mathrm{O}\left({ }^{1} \mathrm{D}\right)$ oxygen state production requires to extend the energy range down to $2 \mathrm{eV}$. This is not necessary for the the $\mathrm{O}\left({ }^{3} \mathrm{~S}\right)$ and $\mathrm{O}\left({ }^{5} \mathrm{~S}\right)$ states, respectively, leading to the UV emission at 130.4 and $135.6 \mathrm{~nm}$ since their electronic impact cross-section cut-off energies are close to $100 \mathrm{eV}$. As reported by Paranicas et al. (1999), there are some concerns about the electron distribution for lower energies.

Since electron densities depend on local plasma properties, we use the UV emission at $135.6 \mathrm{~nm}$ as a proxy for evaluating visible emissions. For auroral regions in the case of Ganymede, UV emissions can reach from 50 to $300 \mathrm{R}$ (see Fig. 3 from Feldman et al. 2000). These latter values are thus used to calibrate our electron distribution. In the case of $300 \mathrm{R}$ for the UV emission, we estimate airlgow emission at $630 \mathrm{~nm}$ to be close to $1 \mathrm{kR}$ for the orbital configuration 2 , i.e. a nadir observation at high latitudes. In the previous section, we have estimated a 
Table 7. Emission estimates at $135.6 \mathrm{~nm}$ and $630 \mathrm{~nm}$, in Rayleigh, induced by electron impact for auroral regions at Ganymede (case 2) for an invariable neutral atmospheric model. The contribution (in $\%$ ) of the photo-induced emission, estimated at $25 \mathrm{R}$ (see Table 6) compared to the total emission at $630 \mathrm{~nm}$, is also indicated.

\begin{tabular}{lcc}
\hline \hline $\mathrm{O}\left({ }^{5} \mathrm{~S}-{ }^{3} \mathrm{P}\right)(135.6 \mathrm{~nm})$ & $\mathrm{O}\left({ }^{1} \mathrm{D}-{ }^{3} \mathrm{P}\right)(630 \mathrm{~nm})$ & Contribution $(\%)$ \\
\hline 50 & 200 & 11 \\
300 & 1000 & 2.4 \\
\hline
\end{tabular}

dayglow of $25 \mathrm{R}$ for high solar conditions (see Table 6), which represents $2.4 \%$ of the emission induced by electron impact only. At high latitude, it is possible to find regions with emission around $50 \mathrm{R}$. It could mean less precipitating electrons or a different atmospheric composition. As stated by Feldman et al. (2000), various combinations of $\mathrm{O}_{2}$ column density and electron temperature are indeed possible. If keeping the same atmospheric model, the $630 \mathrm{~nm}$ emission induced by electron impact represents $200 \mathrm{R}$. In this case, visible emissions from photo-impact represent thus $11 \%$ in the case of Ganymede (see Table 7). These estimations are valid only for auroral regions in the case of Ganymede. In the case of Europa, we can expect a similar photon/electron-induced excitation ratio as a first approximation.

\section{Discussions about the solar UV flux}

The importance of an accurate knowledge of the solar UV flux and its variability for space weather purposes has been previously debated (Lilensten et al. 2008; Barthelemy \& Cessateur 2014). On the basis of several examples, we will show how important an accurate knowledge of solar UV fluxes in terms of dayglow emission modeling is, especially during solar eruptive events. For the particular case of icy satellites, these emissions are not only powerful tools for constraining planetary atmospheres, but are also an important way to infer information regarding the interaction between the satellite and its environment. The impact of solar flares on Europa's atmosphere will first be discussed, for pointing out that Earth-based observations are not always a good solution in order to estimate the solar UV flux throughout the heliosphere. We will discuss some solar UV fluxes, defined as reference spectra, which lead to different dayglow emission estimations.

\subsection{Solar flare events}

In the frame of planetary space weather, the impact of solar flares on dayglow emissions is considered. During such events, the solar flux can increase up to $1000 \%$ for the XUV part, up to $100 \%$ for the EUV spectral range between 20 and $120 \mathrm{~nm}$, and up to $10-30 \%$ for the FUV part between 120 and $200 \mathrm{~nm}$. Both photoionization and photoexcitation processes will thus be directly affected. $4 \mathrm{X}$-class flares are taken into consideration here, listed in Table 8, for which the solar UV flux has been actually measured by TIMED. Figure 10 displays the solar UV flux for the X17 flare occurring on October, 28th 2003. This was one of the most powerful flares ever measured. The solar UV flux for high solar activity $(\mathrm{F} 10.7=195)$ is also displayed for comparison. Earth-based observations are transposed at the Jovian system in order to take center-to-limb position effects into account of solar flares. It is quite well known that flare locations on the solar surface play an important role
Table 8. Red line emission estimations for case 3, for several solar flares events described by their dates and locations on the solar surface as well as their power densities.

\begin{tabular}{lccc}
\hline \hline Events dates & Class & $\begin{array}{c}\text { Location } \\
\text { on solar } \\
\text { surface }\end{array}$ & $\begin{array}{c}\text { Red line } \\
\text { emission (in } \\
\text { Rayleigh) }\end{array}$ \\
\hline January, 15th 2005 & X-1.2 & Center & 409.8 \\
January, 20th 2005 & X-7 & Limb & 411.3 \\
October, 28th 2003 & X-17 & Center & 644.6 \\
November, 4th 2003 & X-17.4 & Limb & 519 \\
\hline F10.7 =195 & - & - & 408.5 \\
\hline
\end{tabular}

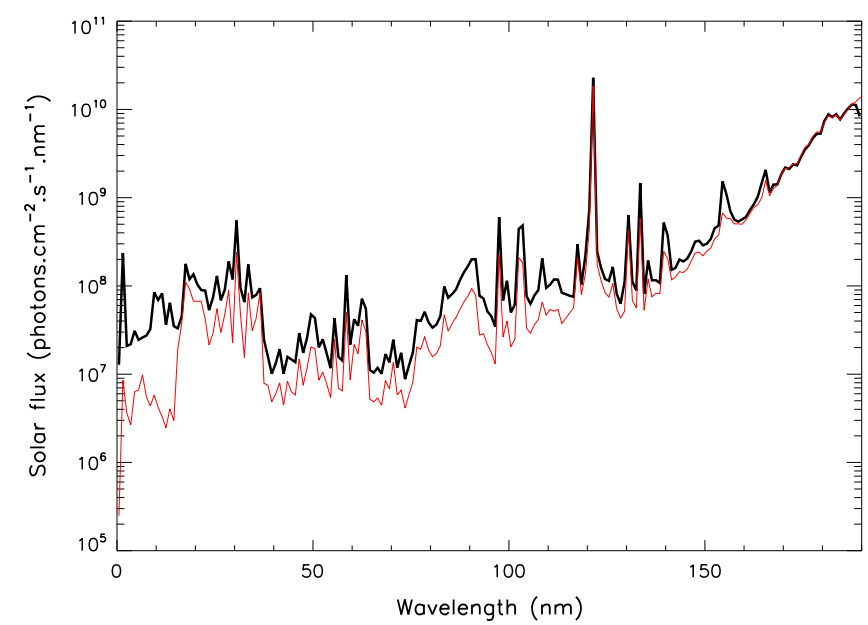

Fig. 10. Solar UV fluxes at the Jovian system, for the X17 flare occurring on October, 28th 2003 (black curve), and for high solar activity conditions $(\mathrm{F} 10.7=195$, red curve $)$.

while looking at the impact on planetary atmospheres, especially in upper atmospheres (Qian et al. 2010). Considering that Jupiter and Earth are very far from opposition at some periods, such an extrapolation can generate some errors in the solar flux estimation.

Since aeronomic emissions are the most important and not polluted by solar reflection from the surface, only case 3 is considered here. Model 1 as neutral atmosphere is used. Table 8 presents our results regarding the different X-class flares for red line emission estimations. For the sake of comparison, estimation for a high level solar activity is also indicated. Not surprisingly, red line emissions increase when considering more powerful flare events. However, there is no simple relationship between the power density of flares and exospheric processes. Two X-17 flares lead to different red line emission and TEC values. Both flares are identical in terms of power density but occur at different locations, one near the disk center and the other near the limb as viewed from the Earth by TIMED. Most emissions in the XUV part of the spectrum $(\lambda \leq 20 \mathrm{~nm})$ are optically thin and not absorbed by the solar atmosphere, while the EUV and FUV parts of the spectrum are optically thick, which leads to a smaller radiation enhancement. Photoproduction of the $\mathrm{O}\left({ }^{1} \mathrm{D}\right)$ state from $\mathrm{O}_{2}$ is mainly induced by solar UV photons from $115 \mathrm{~nm}$ to $177 \mathrm{~nm}$ which are effectively absorbed by the solar atmosphere.

This location effect and the associated radiative transfer effect within the solar atmosphere are even more interesting when considering two flares with different power densities and locations. The X-1.2 flare located close to the disk center 
leads to red line emissions of 409.8 R. An X-7 flare (nearly seven times more powerful in the X-ray domain) located near the limb induces a red line emission of about $411.3 \mathrm{R}$. Flare location is clearly an important factor in determining dayglow emissions for planetary space weather purposes. Using Earthbased measurements for flare events would not be a solution, especially when the Earth-Sun-Planet angle is too important. In the case of planetary space weather, this present study strongly outlines the need for distributed STEREO A \& B like platforms or in-situ observations of the solar UV flux during flare events. For the X-17 solar flare, red line emissions for case 3 are predicted to be of $644.6 \mathrm{R}$, which represents an increased emission compared to quiet high solar conditions by about $58 \%$. The impact of the flare location is again nicely illustrated with the X-17.4 solar flare with a red line estimation of about $519 \mathrm{R}, 20 \%$ less than the X-17 solar flare located at the center of the Sun.

\subsection{Modeling using reference spectra}

Some reference spectra in the UV have been defined for aeronomic purposes. Two spectra, for moderate and high solar activities, are used for inferring rate constants regarding photoabsorption processes for several species (Huebner et al. 1992). Two more recent reference spectra have also been proposed for aeronomic purposes, based on the result of the Atlas mission (Thuillier et al. 2004) for two solar activities $(\mathrm{F} 10.7=78$ and F10.7 = 192). These two spectra will thus be used for assessing dayglow emission, which will be compared to the previous estimations obtained with recent measurements of the solar UV flux. This comparison makes sense since solar activity levels have been chosen to match the Atlas reference spectra ones.

Using both neutral atmospheric models of Smyth \& Marconi (2006) (Model 1) and Shematovich et al. (2005) (Model 2), Table 9 summarizes the estimated red line emissions for observations at the limb. Similar red line emission estimations shall be expected when taking two solar fluxes representing the same level of solar activity into account. There is surprisingly a difference of about $6 \%$. For a high solar activity level, red line emissions are quite variable, from $408.5 \mathrm{R}$ to 433.1 R, in the case of the first neutral atmospheric model. Such a discrepancy is obviously coming from differences within the solar UV flux: the solar Lyman $\alpha$ line as well as the continuum between 130 and $180 \mathrm{~nm}$ are more intense within the Atlas spectrum compared to SORCE/SOLSTICE and TIMED/SEE observations (with a ratio of about 1.12 and 1.02, respectively). Solar indices, such as the F10.7, used here to compare the different solar UV fluxes, actually do not reflect the level of variability in the UV particularly well (Dudok de Wit et al. 2009). Some notable differences between two solar fluxes could thus be expected, even for very similar F10.7 values. Finally, EUV and FUV fluxes are measured for two distinct solar cycles (cycle 22 for Atlas, cycle 23 for TIMED and SORCE observations). Both solar cycles are quite different in terms of irradiance amplitude which could lead to differences within the solar UV flux (Ermolli et al. 2013).

The difference induced by the uncertainties in the solar UV flux might have some implications in planetary atmosphere modeling. Indeed, using two different solar UV fluxes for the same solar conditions as characterized by F10.7 leads to a difference of $6-7 \%$ on the red line emission estimations. The difference arising from using two neutral density profile models leads to an uncertainty of about $14 \%$ for limb observations
Table 9. Red line emission estimations (in Rayleigh) obtained with the Atlas spectrum, compared to those obtained with SORCE and TIMED observations for case 3 .

\begin{tabular}{lcc}
\hline \hline & Model 1 & Model 2 \\
\hline Atlas 1 & 433.1 & 493.6 \\
SORCE/TIMED $(\mathrm{F} 10.7=195)$ & 408.5 & 465 \\
\hline Atlas 3 & 361.5 & 412 \\
SORCE/TIMED $($ F10.7 $=83)$ & 338.3 & 385.9 \\
\hline
\end{tabular}

(see Tables 5 and 9). When considering the solar UV flux impact only, its uncertainties then lead to a difference which is half as important compared to the difference induced by the chosen atmospheric model. However, as a reminder, there is a ratio of about 1.5 for the $\mathrm{O}_{2}$ column density between Model 1 and Model $2\left(\mathrm{~N}_{\mathrm{O}_{2}}^{2} / \mathrm{N}_{\mathrm{O}_{2}}^{1}=1.5\right)$. Let us consider now a hypothetical model 3 , i.e. an average profile $\mathrm{O}_{2}$ density between Model 1 and Model 2, with a ratio of about 1.2 for the $\mathrm{O}_{2}$ column density compared to model $2\left(\mathrm{~N}_{\mathrm{O}_{2}}^{2} / \mathrm{N}_{\mathrm{O}_{2}}^{3}=1.2\right)$. Red line emissions for limb observations at $10 \mathrm{~km}$ above the surface are estimated at $389.6 \mathrm{R}$ using the Atlas 3 spectrum for Model 3. A difference of $1 \%$ then arises between the red line estimation from Model 2 using the solar UV flux from SORCE/TIMED (385.9 R) and Model 3 with the Atlas 3 spectrum (389.6 R). It would then be difficult to distinguish which $\mathrm{O}_{2}$ column density is the most likely between Model 2 and Model 3 (difference of 20\% for the $\mathrm{O}_{2}$ column density) based on our current knowledge of the solar UV flux used as reference spectra.

In regard to our current knowledge of the atmospheric density of the Jovian moons, the uncertainties induced either by the solar UV flux or by the considered atmospheric models are minor (Eviatar et al. 2001; Plainaki et al. 2013, 2015). However, regarding the future JUICE measurements it might be virtually impossible to disentangle atmospheric model errors, even for the regions with no electron precipitation, up to roughly $20 \%$ for the $\mathrm{O}_{2}$ column density because of the solar UV flux uncertainties. This will also necessitate an accurate estimate of the emissions induced by particle precipitation. A proper evaluation of the electron impact-induced excitation leading to the red line emission will be evaluated in a forthcoming paper, using a transport model within Europa and Ganymede atmospheres.

Previous results suggest that we need accurate solar data, and this for the whole EUV/FUV part of the spectrum. For doing so, special efforts have to be made to assess the absolute values of the solar UV flux along its intrinsic variability for space weather purposes. Using reference spectra such as Atlas is not a recommended solution as previously illustrated. One other alternative would be to rely on solar UV models. Major efforts have been done toward developing robust models, based on solar proxies, such as the radio flux at $10.7 \mathrm{~cm}$ (F10.7) (Tapping \& Detracey 1990) or the MgII core-to-wing index (Heath \& Schlesinger 1986), which should assess the solar UV flux variability. However, it has been largely argued that no combination of existing indices can properly reconstruct the solar EUV/FUV irradiance on all time scales (see e.g. Dudok de Wit et al. 2009; Cessateur et al. 2011). This is quite prejudicial for properly characterizing dayglow emissions as previously seen, and more generally for retrieving upper atmosphere parameters (see e.g. Lilensten et al. 2007; Cessateur et al. 2012a). There is then no better alternative than direct measurements of the local solar UV flux in near-real time. 
We then advocate that any planetary missions should embark on some instruments devoted to the characterization of the solar UV flux. This would certainly remove one part of the uncertainties within modeling processes. Cessateur et al. (2012b) have proposed an instrumental concept, particularly suited for planetary missions. With a low weight and using radiation-hard materials, a radiometer composed of five spectral bands can provide the solar UV flux with great accuracy, but for a non-flaring Sun. Further developments are clearly needed for assessing the solar UV flux during flare events with simple instruments such as radiometers. This instrument is suited not only in the case of a Jovian mission for assessing Europa's and Ganymede's atmospheres, but clearly for all planetary missions, where dayglow emissions are measured.

\section{Conclusions}

We have explored in this paper the effects of the solar UV flux and its variability on the dayglow of Europa and Ganymede. The thermal electron population, as well as the photochemical production rates along the atmosphere of different oxygen states, namely $\mathrm{O}\left({ }^{3} \mathrm{P}\right), \mathrm{O}\left({ }^{1} \mathrm{D}\right)$, and $\mathrm{O}\left({ }^{1} \mathrm{~S}\right)$, are the outcomes of the modeling. The continuity equation within the atmosphere of both moons has been solved in order to calculate the number densities of the excited states. Considering several orbital configurations, dayglow emission induced by solar UV photons only has been subsequently estimated for the red and green lines at $630 \mathrm{~nm}$ and $577.7 \mathrm{~nm}$, respectively. Taking the velocity as modeled by Shematovich et al. (2005) and Marconi (2007) into account, the transport is a critical process with a significant impact on the radial distribution of $\mathrm{O}\left({ }^{1} \mathrm{D}\right)$. As orbital configurations, we considered limb observations $10 \mathrm{~km}$ above the surface, and red line emissions have been estimated around $400 \mathrm{R}$ for high solar conditions, using the neutral atmospheric model of Smyth \& Marconi (2006). Those emissions, induced by photo-impact only, are however a secondary process compared to electron-impact excitation and can contribute few percent of the total visible emission in auroral regions of Ganymede.

Using planetary atmospheric emissions for assessing both atmospheric compositions and energetic inputs is a highly degenerate case study, especially within regions where both electron precipitation and the solar UV flux are responsible for the dissociation and excitation of the neutral species. A way to reduce the degeneracy of the system is then to separate electron and the solar UV flux problems. The importance of an accurate knowledge of the solar UV flux and its variability for space weather purposes has also been discussed. The impact of the solar flares on dayglow emission from Europa has been explored for the first time. Location effects of solar flares have been enlightened, which supports the idea that in-situ observations of the solar UV flux are mandatory when performing planetary space weather studies. Using reference spectra might also lead to different dayglow emission estimations, compared to the ones obtained with recent solar UV measurements for the same level of solar activity. This difference of about $6 \%$ might affect planetary atmosphere modeling. For regions without electronic precipitation, this could also prevent the discrimination of two distinctive neutral atmospheric models if their $\mathrm{O}_{2}$ column densities differ by roughly $20 \%$, when only using red line emissions observations.

This present study makes sense when considering the future JUICE mission outcomes. Indeed, in-situ measurement will provide better neutral and electron atmospheric densities and secondary processes as photo impact-induced ionization and excitation could be of importance to correctly interpret the data. In order to do so, a necessary step will be to disentangle the different contributions, electronic from photo processes. Moreover, the variability of the solar UV flux, from quiet conditions to transient events such as solar flares, can lead to important red line emission variability at limb viewing. The difference, induced by the solar UV flux, could impact the resulting planetary atmosphere models, which outlines the need for direct measurements of the solar UV flux.

Acknowledgements. GC was supported by the Fonds de la Recherche Scientifique Grant PDR T.1073.14 Comparative study of atmospheric erosion and received funding from the European Community's Seventh Framework Programme (FP7/2007-2013) under Grant Agreement No. 284461 (eHEROES project, http:/ soteria-space.eu/eheroes $/ \mathrm{html} /$ ) and No. 313188 (SOLID project, https://projects.pmodwrc.ch/solid/). GC also cknowledges support from Swiss COST office (Grant No. C11.0135). The authors and the editor thank two anonymous referees for their assistance in evaluating this paper.

\section{References}

Atkinson, R., D.L. Baulch, R.A. Cox, J.N. Crowley, R.F. Hampson, R.G. Hynes, M.E. Jenkin, M.J. Rossi, and J. Troe. Evaluated kinetic and photochemical data for atmospheric chemistry: volume $\mathrm{I}-$ gas phase reactions of $\mathrm{O}_{x} . \mathrm{HO}_{x}, \mathrm{NO}_{x}$ and $\mathrm{SO}_{x}$ species. Atmos. Chem. Phys., 4 (6), 1461-1738, 2004, DOI: $10.5194 /$ acp-4-1461-2004.

Banks, P., and G. Kockarts. Aeronomy, Part A, Academic Press, New York, 1973, DOI: 10.1016/B978-0-12-077802-7.50002-6.

Barthelemy, M., and G. Cessateur. Sensitivity of upper atmospheric emissions calculations to solar/stellar UV flux. J. Space Weather Space Clim., 4 (27), A35, 2014, DOI: 10.1051/swsc/2014033.

Calvin, W.M., R.N. Clark, R.H. Brown, and J.R. Spencer. Spectra of the icy Galilean satellites from 0.2 to $5 \mu \mathrm{m}$ : a compilation, new observations, and a recent summary. J. Geophys. Res., 100 , 19041-19048, 1995, DOI: 10.1029/94JE03349.

Cassidy, T.A., R.E. Johnson, M.A. McGrath, M.C. Wong, and J.F. Cooper. The spatial morphology of Europa's near-surface $\mathrm{O}_{2}$ atmosphere. Icarus, 191, 755-764, 2007,

DOI: $10.1016 /$ j.icarus.2007.04.033.

Cassidy, T.A., R.E. Johnson, P.E. Geissler, and F. Leblanc. Simulation of $\mathrm{Na} D$ emission near Europa during eclipse. J. Geophys. Res. [Planets], 113, E02005, 2008,

DOI: 10.1029/2007JE002955.

Cessateur, G., T. Dudok de Wit, M. Kretzschmar, J. Lilensten, J. Hochedez, and M. Snow. Monitoring the solar UV irradiance spectrum from the observation of a few passbands. $A \& A, \mathbf{5 2 8}$, A68, 2011, DOI: 10.1051/0004-6361/201015903.

Cessateur, G., J. Lilensten, M. Barthélémy, T. Dudok de Wit, C. Simon Wedlund, G. Gronoff, H. Ménager, and M. Kretzschmar. Photoabsorption in Ganymede's atmosphere. Icarus, 218, 308-319, 2012a, DOI: 10.1016/j.icarus.2011.11.025.

Cessateur, G., J. Lilensten, T. Dudok de Wit, A. BenMoussa, and M. Kretzschmar. New observation strategies for the solar UV spectral irradiance. J. Space Weather Space Clim., 2, A16, 2012b, DOI: $10.1051 /$ swsc/2012016.

Dudok de Wit, T., M. Kretzschmar, J. Lilensten, and T. Woods. Finding the best proxies for the solar UV irradiance. Geophys. Res. Lett., 36, L10107, 2009, DOI: 10.1029/2009GL037825.

Ermolli, I., K. Matthes, T. Dudok de Wit, N.A. Krivova, K. Tourpali, et al. Recent variability of the solar spectral irradiance and its impact on climate modelling. Atmos. Chem. Phys., 13, 3945-3977, 2013, DOI: 10.5194/acp-13-3945-2013.

Eviatar, A., D.F. Strobel, B.C. Wolven, P.D. Feldman, M.A. McGrath, and D.J. Williams. Excitation of the Ganymede ultraviolet aurora. Astrophys. J., 555, 1013-1019, 2001, DOI: $10.1086 / 321510$. 
Feldman, P.D., M.A. McGrath, D.F. Strobel, H.W. Moos, K.D. Retherford, and B.C. Wolven. HST/STIS ultraviolet imaging of polar aurora on Ganymede. Astrophys. J., 535, 1085-1090, 2000, DOI: $10.1086 / 308889$.

Fischer, C.F., and G. Tachiev. Breit-Pauli energy levels, lifetimes, and transition probabilities for the beryllium-like to neon-like sequences. At. Data Nucl. Data Tables, 87 (1), 1-184, 2004, DOI: 10.1016/j.adt.2004.02.001

Florescu-Mitchell, A.I., and J.B.A. Mitchell. Dissociative recombination. Phys. Rep., 430, 277-374, 2006, DOI: $10.1016 /$ j.physrep.2006.04.002.

Grasset, O., M.K. Dougherty, A. Coustenis, E.J. Bunce, C. Erd, et al. JUpiter ICy moons Explorer (JUICE): an ESA mission to orbit Ganymede and to characterise the Jupiter system. Planet. Space Sci., 78, 1-21, 2013, DOI: 10.1016/j.pss.2012.12.002.

Gronoff, G., C. Simon Wedlund, C.J. Mertens, and R.J. Lillis. Computing uncertainties in ionosphere-airglow models: I. Electron flux and species production uncertainties for Mars. J. Geophys. Res. [Space Phys.], 117, A04306, 2012, DOI: $10.1029 / 2011$ JA016930.

Gurnett, D.A., W.S. Kurth, A. Roux, S.J. Bolton, and C.F. Kennel. Evidence for a magnetosphere at Ganymede from plasma-wave observations by the Galileo spacecraft. Nature, 384, 535-537, 1996, DOI: $10.1038 / 384535 \mathrm{a} 0$.

Hall, D.T., P.D. Feldman, M.A. McGrath, and D.F. Strobel. The farultraviolet oxygen airglow of Europa and Ganymede. Astrophys. J., 499, 475-481, 1998, DOI: 10.1086/305604.

Heath, D.F., and B.M. Schlesinger. The Mg 280-nm doublet as a monitor of changes in solar ultraviolet irradiance. J. Geophys. Res., 91, 8672-8682, 1986, DOI: 10.1029/JD091iD08p08672.

Huebner, W.F., J.J. Keady, and S.P. Lyon. Solar photo rates for planetary atmospheres and atmospheric pollutants. Astrophys. Space Sci., 195, 1-289, 1992, DOI: 10.1007/BF00644558.

Jackman, C.M., and C.S. Arridge. Solar cycle effects on the dynamics of Jupiter's and Saturn's magnetospheres. Sol. Phys., 274, 481-502, 2011, DOI: 10.1007/s11207-011-9748-z.

Kalogerakis, K.S., T.G. Slanger, E.A. Kendall, T.R. Pedersen, M.J. Kosch, B. Gustavsson, and M.T. Rietveld. Remote Oxygen Sensing by Ionospheric Excitation (ROSIE). Ann. Geophys., 27 (5), 2183-2189, 2009, DOI: 10.5194/angeo-27-2183-2009.

Kella, D., L. Vejby-Christensen, P. Johnson, H. Pedersen, and L. Andersen. The source of green light emission determined from a heavy-ion storage ring experiment. Science, 276, 1530-1533, 1997.

Kivelson, M.G., K.K. Khurana, C.T. Russell, R.J. Walker, J. Warnecke, F.V. Coroniti, C. Polanskey, D.J. Southwood, and G. Schubert. Discovery of Ganymede's magnetic field by the Galileo spacecraft. Nature, 384, 537-541, 1996, DOI: $10.1038 / 384537 \mathrm{a} 0$.

Kliore, A.J., D.P. Hinson, F.M. Flasar, A.F. Nagy, and T.E. Cravens. The ionosphere of Europa from Galileo radio occultations. Science, 277, 355-358, 1997, DOI: $10.1126 /$ science. 277.5324 .355$.

Lilensten, J., T. Dudok de Wit, P.-O. Amblard, J. Aboudarham, F. Auchre, and M. Kretzschmar. Recommendation for a set of solar EUV lines to be monitored for aeronomy applications. Ann. Geophys., 25 (6), 1299-1310, 2007. DOI: 10.5194/angeo-25-1299-2007.

Lilensten, J., T. Dudok de Wit, M. Kretzschmar, P. Amblard, S. Moussaoui, J. Aboudarham, and F. Auchère. Review on the solar spectral variability in the EUV for space weather purposes. Ann. Geophys., 26, 269-279, 2008, DOI: 10.5194/angeo-26-269-2008.

Lilensten, J., A.J. Coates, V. Dehant, T. Dudok de Wit, R.B. Horne, F. Leblanc, J. Luhmann, E. Woodfield, and M. Barthélemy. What characterizes planetary space weather? Astron. Astrophys. Rev., 22, 79, 2014, DOI: 10.1007/s00159-014-0079-6.

Marconi, M.L. A kinetic model of Ganymede's atmosphere, Icarus, 190, 155-174, 2007, DOI: 10.1016/j.icarus.2007.02.016.

McGrath, M.A., X. Jia, K. Retherford, P.D. Feldman, D.F. Strobel, and J. Saur. Aurora on Ganymede. J. Geophys. Res. [Space Phys.], 118, 2043-2054, 2013, DOI: 10.1002/jgra.50122.
Nagy, A.F., J. Kim, T.E. Cravens, and A.J. Kliore. Hot oxygen corona at Europa. Geophys. Res. Lett., 25, 4153-4155, 1998, DOI: 10.1029/1998GL900139.

Paranicas, C., W.R. Paterson, A.F. Cheng, B.H. Mauk, R.W. McEntire, L.A. Frank, and D.J. Williams. Energetic particle observations near Ganymede. J. Geophys. Res., 104, 17459-17470, 1999, DOI: 10.1029/1999JA900199.

Pavlov, A., N. Pavlova, and A. Drozdov. Production rate of $\mathrm{O}\left({ }^{1} \mathrm{D}\right)$, $\mathrm{O}\left({ }^{1} \mathrm{~S}\right)$ and $\mathrm{N}\left({ }^{2} \mathrm{D}\right)$ in the subauroral red arc region. Geomag. Aeron., 39, 201205, 1999.

Payan, A.P., C.S. Paty, and K.D. Retherford. Uncovering local magnetospheric processes governing the morphology and variability of Ganymede's aurora using three-dimensional multifluid simulations of Ganymede's magnetosphere. J. Geophys. Res. [Space Phys.], 120, 401-413, 2015, DOI: $10.1002 / 2014 J A 020301$.

Plainaki, C., A. Milillo, A. Mura, S. Orsini, and T. Cassidy. Neutral particle release from Europa's surface. Icarus, 210, 385-395, 2010, DOI: $10.1016 /$ j.icarus.2010.06.041.

Plainaki, C., A. Milillo, A. Mura, J. Saur, S. Orsini, and S. Massetti. Exospheric $\mathrm{O}_{2}$ densities at Europa during different orbital phases. Planet. Space Sci., 88, 42-52, 2013, DOI: $10.1016 /$ j.pss.2013.08.011.

Plainaki, C., A. Milillo, S. Massetti, A. Mura, X. Jia, S. Orsini, V. Mangano, E. De Angelis, and R. Rispoli. The $\mathrm{H}_{2} \mathrm{O}$ and $\mathrm{O}_{2}$ exospheres of Ganymede: the result of a complex interaction between the Jovian magnetospheric ions and the icy moon. Icarus, 245, 306-319, 2015, DOI: 10.1016/j.icarus.2014.09.018.

Porco, C.C., R.A. West, A. McEwen, A.D. Del Genio, A.P. Ingersoll, et al. Cassini imaging of Jupiter's atmosphere, satellites, and rings. Science, 299, 1541-1547, 2003,

DOI: $10.1126 /$ science. 1079462

Qian, L., A.G. Burns, P.C. Chamberlin, and S.C. Solomon. Flare location on the solar disk: modeling the thermosphere and ionosphere response. J. Geophys. Res. [Space Phys.], 115 (A9), A09311, 2010, DOI: 10.1029/2009JA015225.

Rosen, S., A. Derkatch, J. Semaniak, A. Neau, A. Al-Khalili, et al. Recombination of simple molecular ions studied in storage ring: dissociative recombination of $\mathrm{H}_{2} \mathrm{O}^{+}$. Faraday Discussion, 115, 295-302, 2000, DOI: 10.1039/A909314A.

Roth, L., J. Saur, K.D. Retherford, D.F. Strobel, P.D. Feldman, M.A. McGrath, and F. Nimmo. Transient water vapor at Europa's south pole. Science, 343, 171-174, 2014, DOI: $10.1126 /$ science. 1247051 .

Rottman, G. The SORCE mission. Sol. Phys., 230, 7-25, 2005, DOI: $10.1007 / \mathrm{s} 11207-005-8112-6$.

Saur, J., D.F. Strobel, and F.M. Neubauer. Interaction of the Jovian magnetosphere with Europa: constraints on the neutral atmosphere. J. Geophys. Res., 103, 19947-19962, 1998 , DOI: 10.1029/97JE03556.

Saur, J., S. Duling, L. Roth, X. Jia, D.F. Strobel, et al. The search for a subsurface ocean in Ganymede with Hubble Space Telescope observations of its auroral ovals. J. Geophys. Res. [Space Phys.], 120, 1715-1737, 2015, DOI: 10.1002/2014JA020778.

Schmidt, H., R. Wegmann, W. Huebner, and D. Boice. Cometary gas and plasma flow with detailed chemistry. Comput. Phys. Commun., 49 (1), 17-59, 1988, DOI: 10.1016/0010-4655(88)90214-7.

Shematovich, V.I., R.E. Johnson, J.F. Cooper, and M.C. Wong. Surface-bounded atmosphere of Europa, Icarus, 173, 480-498, 2005, DOI: $10.1016 /$ j.icarus.2004.08.013.

Slanger, T.G., P.C. Cosby, B.D. Sharpee, K.R. Minschwaner, and D.E. Siskind. $\mathrm{O}\left({ }^{1} \mathrm{~S} \rightarrow{ }^{1} \mathrm{D},{ }^{3} \mathrm{P}\right)$ branching ratio as measured in the terrestrial nightglow. J. Geophys. Res. [Space Phys.], 111, A12318, 2006, DOI: 10.1029/2006JA011972.

Smyth, W.H., and M.L. Marconi. Europa's atmosphere, gas tori, and magnetospheric implications. Icarus, 181, 510-526, 2006, DOI: 10.1016/j.icarus.2005.10.019.

Stuhl, F., and K.H. Welge. Deactivation of $\mathrm{O}\left({ }^{1} \mathrm{~S}\right)$ and $\mathrm{O}_{2}\left(\mathrm{~b}^{1} \Sigma_{\mathrm{g}}{ }^{+}\right)$. Can. J. Chem., 47 (10), 1870-1877, 1969,

DOI: $10.1139 / \mathrm{v} 69-306$. 
Tapping, K.F., and B. Detracey. The origin of the $10.7 \mathrm{~cm}$ flux. Sol. Phys., 127, 321-332, 1990, DOI: 10.1007/BF00152171.

Thirupathaiah, P., and V. Singh. An updated model of atomic oxygen redline dayglow emission. Adv. Space Res., 54 (6), 939-945, 2014, DOI: 10.1016/j.asr.2014.05.022.

Thuillier, G., L. Floyd, T.N. Woods, R. Cebula, E. Hilsenrath, M. Hersé, and D. Labs. Solar Irradiance Reference Spectra. In: J.M. Pap, et al., Editors. Solar Variability and its Effects on Climate. Geophysical, Vol. 141 of Washington DC American Geophysical Union Geophysical Monograph Series, 171, 2004, DOI: $10.1029 / \mathrm{GM} 141$.

Turc, L., L. Leclercq, F. Leblanc, R. Modolo, and J.-Y. Chaufray. Modelling Ganymede's neutral environment: a 3D test-particle simulation. Icarus, 229, 157-169, 2014, DOI: $10.1016 /$ j.icarus.2013.11.005.

Wiese, W.L., J.R. Fuhr, and T.M. Deters. Atomic transition probabilities of carbon, nitrogen, and oxygen: a critical data compilation. Monograph No. 7 of the Journal of Physical and Chemical Reference Data, American Chemical Society for the
National Institute of Standards and Technology, Washington DC, ISBN: 1-56396-602-6, 1996.

Witasse, O., J. Lilensten, C. Lathuillère, and P.-L. Blelly. Modeling the OI 630.0 and $557.7 \mathrm{~nm}$ thermospheric dayglow during EISCAT-WINDII coordinated measurements. J. Geophys. Res., 104, 24639-24656, 1999, DOI: 10.1029/1999JA900260.

Woods, T.N., F.G. Eparvier, S.M. Bailey, P.C. Chamberlin, J. Lean, G.J. Rottman, S.C. Solomon, W.K. Tobiska, and D.L. Woodraska. Solar EUV Experiment (SEE): mission overview and first results. J. Geophys. Res. [Space Phys.], 110, A01312, 2005, DOI: $10.1029 / 2004 J A 010765$.

Woods, T.N., P.C. Chamberlin, W.K. Peterson, R.R. Meier, P.G. Richards, et al. XUV Photometer System (XPS): improved solar irradiance algorithm using CHIANTI spectral models. Sol. Phys., 250, 235-267, 2008.

Zipf, E.C. The collisional deactivation of metastable atoms and molecules in the upper atmosphere. Can. J. Chem., 47 (10), 1863-1870, 1969, DOI: 10.1139/v69-305.

Cite this article as: Cessateur G, Barthelemy M \& Peinke I. Photochemistry-emission coupled model for Europa and Ganymede. J. Space Weather Space Clim., 6, A17, 2016, DOI: 10.1051/swsc/2016009. 